TRANSACTIONS OF THE

AMERICAN MATHEMATICAL SOCIETY

Volume 364, Number 6, June 2012, Pages 3217-3239

S 0002-9947(2012)05508-5

Article electronically published on February 3, 2012

\title{
VECTOR BUNDLES OVER DAVIS-JANUSZKIEWICZ SPACES WITH PRESCRIBED CHARACTERISTIC CLASSES
}

\author{
DIETRICH NOTBOHM
}

\begin{abstract}
For any $(n-1)$-dimensional simplicial complex, we construct a particular $n$-dimensional complex vector bundle over the associated DavisJanuszkiewicz space whose Chern classes are given by the elementary symmetric polynomials in the generators of the Stanley Reisner algebra. We show that the isomorphism type of this complex vector bundle as well as of its realification are completely determined by its characteristic classes. This allows us to show that coloring properties of the simplicial complex are reflected by splitting properties of this bundle and vice versa. Similar questions are also discussed for $2 n$-dimensional real vector bundles with particular prescribed characteristic Pontrjagin and Euler classes. We also analyze which of these bundles admit a complex structure. It turns out that all these bundles are closely related to the tangent bundles of quasi-toric manifolds and moment angle complexes.
\end{abstract}

\section{INTRODUCTION}

For any finite simplicial complex $K$, Davis and Januszkiewicz constructed a family of realizations of the Stanley-Reisner algebra $\mathbb{Z}[K]$, that is, the integral cohomology of these spaces is isomorphic to $\mathbb{Z}[K]$. They also showed that all these spaces are homotopy equivalent [DJ, Section 4]. We denote a generic model for this homotopy type by $D J(K)$. In Section 6 of the above-mentioned paper, Davis and Januszkiewicz also studied particular vector bundles over $D J(K)$. They constructed an $m$-dimensional complex bundle $\lambda \downarrow D J(K)$ whose Chern classes are given by the elementary symmetric polynomials in the generators of the Stanley Reisner algebra $\mathbb{Z}[K]$ and compared it with vector bundles obtained from the tangent bundles of the moment angle complex or a quasi-toric manifold by an application of the Borel construction. In particular, they showed that these bundles are stably isomorphic and have the same Pontrjagin classes. We were wondering to which extent the characteristic classes determine the isomorphism type of these bundles.

We will split off a large trivial vector bundle from $\lambda$ and will show that the isomorphism type of the remaining vector bundle $\xi \downarrow D J(K)$ as well as of its realification $\xi_{\mathbb{R}}$ is completely determined by its characteristic classes. We will also study real vector bundles $\rho$ with the same Pontrjagin classes as $\xi$ and prove for them similar existence and uniqueness results in terms of Pontrjagin and Euler classes. We are able to offer several applications of the uniqueness results: Colorings of $K$ are reflected by (stable) splittings of $\xi$ and $\xi_{\mathbb{R}}$ into a direct sum of complex

Received by the editors June 25, 2009 and, in revised form, November 18, 2010.

2010 Mathematics Subject Classification. Primary 55R25, 57R22, 05 C15.

Key words and phrases. Davis-Januszkiewicz space, vector bundle, characteristic classes, coloring, simplicial complex, complex structure.

(C)2012 American Mathematical Society 3217 
line bundles or of 2-dimensional real bundles. We will also improve on the stable isomorphisms results mentioned above and classify complex structures on the real vector bundles $\rho$.

To make our statements more precise we need to fix notation and to recall some basic constructions. An abstract simplicial complex on the finite vertex set $V$ of order $|V|=m$ is a set $K=\left\{\alpha_{1}, \ldots, \alpha_{s}\right\}$ of subsets $\alpha_{i} \subset V$ which is closed under the formation of subsets and which contains the empty set $\emptyset$. We will always identify $V$ with the set $[m]:=\{1, \ldots, m\}$ of the first $m$ natural numbers. The dimension $\operatorname{dim} K$ of $K$ is expressed in terms of the cardinality of its faces $\alpha \in K$. We set $\operatorname{dim} \alpha:=|\alpha|-1$, and $\operatorname{dim} K$ is the maximum of the dimensions of its faces. Some of our statements involve sums taken over all maximal faces. We will denote this set by $M_{K}$.

Examples are given by full simplices and their boundaries. For a set $\alpha$ we denote by $\Delta[\alpha]$ the simplicial complex given by all subsets of $\alpha$ and by $\partial \Delta[\alpha]$ the complex of all proper subsets of $\alpha$. Then $\operatorname{dim} \Delta[\alpha]=|\alpha|-1$ and $\operatorname{dim} \partial \Delta[\alpha]=|\alpha|-2$. The set $M_{\Delta[\alpha]}$ consists only of the set $\alpha$ and $M_{\partial \Delta[\alpha]}$ consists of all subsets of $\alpha$ of order $|\alpha|-1$. The complex $K$ is a subcomplex of the full simplex $\Delta[m]$ on $m$-vertices, and for each $\alpha \in K$, the complex $\Delta[\alpha] \subset K$ is a subcomplex of $K$.

For a commutative ring $R$ with unit we denote by $R[m]:=R\left[v_{1}, \ldots, v_{m}\right]$ the graded polynomial algebra generated by algebraically independent elements $v_{1}, \ldots, v_{m}$ of degree 2, one for each vertex of $K$. For each subset $\alpha \subseteq[m]$ we denote by $v_{\alpha}:=$ $\prod_{j \in \alpha} v_{j}$ the square free monomial whose factors are in one-to-one correspondence with vertices contained in $\alpha$. The graded Stanley-Reisner algebra $R[K]$ associated with $K$ is defined as the quotient $R[K]:=R[m] / I_{K}$, where $I_{K} \subset R[m]$ is the ideal generated by all elements $v_{\beta}$ such that $\beta \subseteq[m]$ is not a face of $K$.

For a complex vector bundle $\eta$ over a space $X$, we denote by $c_{i}(\eta) \in H^{2 i}(X ; \mathbb{Z})$ the $i$-th Chern class and by $c(\eta):=1+\Sigma_{i \geq 1} c_{i}(\eta)$ the total Chern class of $\eta$. For a real vector bundle $\rho$ over $X$, we use $p_{i}(\rho) \in H^{4 i}(X ; \mathbb{Z})$ and $p(\rho):=1+\Sigma_{i \geq 1} p_{i}(\rho)$ to denote the $i$-th and the total Pontrjagin class of $\rho$. If $\rho$ is $k$-dimensional and oriented, the Euler class is denoted by $e(\rho) \in H^{k}(X ; \mathbb{Z})$.

We fix an isomorphism $H^{*}(D J(K) ; \mathbb{Z}) \cong \mathbb{Z}[K]$. Since $B T^{m}$ is an EilenbergMac Lane space realizing the algebra $\mathbb{Z}[m]$, there exists a map $q_{K}: D J(K) \longrightarrow B T^{m}$ which induces in cohomology the projection $\mathbb{Z}[m] \longrightarrow \mathbb{Z}[K]$. We can think of $T^{m}$ as the maximal torus $T^{m} \subset U(m)$. The pull back of the $m$-dimensional universal complex vector bundle over $B U(m)$ along the composition of $q_{K}$ with the maximal torus inclusion $B T^{m} \longrightarrow B U(m)$ produces a vector bundle $\lambda \downarrow D J(K)$ whose total Chern class is $c(\lambda)=\prod_{i=1}^{m}\left(1+v_{i}\right)$. Also, the total Pontrjagin class of the realification $\lambda_{\mathbb{R}}$ is $p\left(\lambda_{\mathbb{R}}\right)=\prod_{i=1}^{m}\left(1-v_{i}^{2}\right)$ [DJ, Section 6]. These characteristic classes play a particular role in our statements, and we define $c(K):=\prod_{i=1}^{m}\left(1+v_{i}\right)$ and $p(K):=\prod_{i=1}^{m}\left(1-v_{i}^{2}\right)$.

Confusing notation we also denote by $\mathbb{C}$ and $\mathbb{R} 1$-dimensional trivial complex or real vector bundles over a space $X$.

Theorem 1.1. Let $K$ be an $(n-1)$-dimensional finite simplicial complex. Then there exists an $n$-dimensional complex vector bundle $\xi$ over $D J(K)$ such that $\lambda \cong$ $\xi \oplus \mathbb{C}^{m-n}$. In particular, $c(\xi)=c(K)$ and $p\left(\xi_{\mathbb{R}}\right)=p(K)$. Moreover, $c_{n}(\xi)$ and $p_{n}\left(\xi_{\mathbb{R}}\right)$ are non-trivial.

The vector bundle $\xi \downarrow D J(K)$ also satisfies uniqueness properties. Its isomorphism type is completely determined by its characteristic classes. The realification 
$\xi_{\mathbb{R}}$ of a complex bundle $\xi$ carries a canonical orientation. Also, $\xi_{\mathbb{R}}$ denotes the underlying oriented real vector bundle with this canonical orientation. The Euler class $e\left(\xi_{\mathbb{R}}\right)$ is then given by the $n$-th Chern class $c_{n}(\xi)$. Trivial real vector bundles get the standard orientation.

Theorem 1.2. Let $K$ be an $(n-1)$-dimensional finite simplicial complex. Let $\eta \downarrow D J(K)$ be an $r$-dimensional complex and $\rho \downarrow D J(K)$ an oriented s-dimensional real vector bundle over $D J(K)$.

(i) If $c(\eta)=c(\xi)$, then $r \geq n$ and $\eta$ and $\xi \oplus \mathbb{C}^{r-n}$ are isomorphic.

(ii) If $p(\rho)=p\left(\xi_{\mathbb{R}}\right)$, then $s \geq 2 n$. If $s>2 n$, then $\rho$ and $\xi_{\mathbb{R}} \oplus \mathbb{R}^{s-2 n}$ are isomorphic. Also, if $s=2 n$, then $\rho \oplus \mathbb{R}$ and $\xi_{\mathbb{R}} \oplus \mathbb{R}$ are isomorphic.

(iii) If $s=2 n, p(\rho)=p\left(\xi_{\mathbb{R}}\right)$ and $e(\rho)=e\left(\xi_{\mathbb{R}}\right)$, then $\rho$ and $\xi_{\mathbb{R}}$ are isomorphic.

For clarification, the isomorphisms in parts (ii) and (iii) are isomorphisms of oriented bundles.

In Section 4 we will allow more general Euler classes. A function $\omega: M_{K} \longrightarrow$ $\{ \pm 1\}$ gives rise to class $e_{\omega}(K):=\sum_{\mu \in M_{K}} \omega(\mu) v_{\mu} \in H^{2 n}(D J(K) ; \mathbb{Z})$. For each function $\omega$ we will construct an oriented real vector bundle $\rho_{\omega}$ over $\operatorname{DJ}(K)$ with $e\left(\rho_{\omega}\right)=e_{\omega}(K)$ and $p\left(\rho_{\omega}\right)=p(K)$ (Theorem 4.2). The bundles $\rho_{\omega}$ also satisfy uniqueness results in terms of Euler and Pontrjagin classes (Theorem 4.3) and provide a complete list of isomorphism types of (oriented) $2 n$-dimensional real vector bundles whose total Pontrjagin class equals $\prod_{i \in[m]}\left(1-v_{i}^{2}\right)$.

Theorem 1.3. Let $K$ be an $(n-1)$-dimensional finite simplicial complex. Let $\rho \downarrow D J(K)$ be an oriented $2 n$-dimensional real vector bundle such that $p(\rho)=$ $\prod_{i \in[m]}\left(1-v_{i}^{2}\right)$. Then there exists a function $\omega: M_{K} \longrightarrow\{ \pm 1\}$ such that $\rho$ and $\rho_{\omega}$ are isomorphic.

The realification $\xi_{\mathbb{R}}$ satisfies the assumption of the last theorem, realizes the function $\omega \equiv 1$, i.e. $\omega(\mu)=1$ for all $\mu \in M_{K}$, and obviously admits a complex structure. We say that a real vector bundle $\rho$ admits a complex structure if there exists a complex bundle $\eta$ such that $\rho$ and $\eta_{\mathbb{R}}$ are isomorphic as non-oriented real vector bundles. For a function $f:[m] \longrightarrow\{ \pm 1\}$ we define a function $\omega_{f}: M_{K} \longrightarrow$ $\{ \pm 1\}$ by $\omega_{f}(\mu):=\prod_{i \in \mu} f(i)$ and denote $e_{\omega_{f}}(K)$ by $e_{f}(K)$.

Theorem 1.4. Let $K$ be an $(n-1)$-dimensional finite simplicial complex. Let $\rho$ be a $2 n$-dimensional oriented real vector bundle over $D J(K)$ such that $p(\rho)=p(K)$. Then, the bundle $\rho$ admits a complex structure if and only if there exists a function $f:[m] \longrightarrow\{ \pm 1\}$ such that $e(\rho)= \pm e_{f}(K)$.

Remark 1.5. Since Davis-Januszkiewicz spaces are simply connected, every real vector bundle $\rho$ over $D J(K)$ is orientable. The Euler classes for different orientations may only differ by a sign. In Theorem 1.2 , Theorem 1.3 and Theorem 1.4 we made the assumption that $\rho$ is oriented. This is not a serious restriction. For example, if $\eta$ is a complex and $\rho$ a real vector bundle over $D J(K)$ such that $\rho$ and $\eta_{\mathbb{R}}$ are isomorphic as non-oriented vector bundles, then the canonical orientation on $\eta_{\mathbb{R}}$ must agree with one of the possible orientations for $\rho$. That is, that $\xi_{\mathbb{R}}$ and $\rho$ with the appropriate orientation are isomorphic as oriented bundles.

Several of the vector bundles will be constructed as homotopy orbit spaces. For a compact Lie group $G$ and a $G$-space $X$, the Borel construction or homotopy orbit space $E G \times_{G} X$ will be denoted by $X_{h G}$. If $\eta$ is an $n$-dimensional $G$-vector bundle 
over $X$ with total space $E(\eta)$, the Borel construction establishes a fiber bundle $E(\eta)_{h G} \longrightarrow X_{h G}$. In fact, this is an $n$-dimensional vector bundle over $X_{h G}[\underline{\mathrm{S}}$, denoted by $\eta_{h G}$.

There are several connections with the work of Davis and Januszkiewicz, in particular with Section 6 of [DJ]. For every simplicial complex $K$ there exists an associated moment angle complex $Z_{K}$ which carries a $T^{m}$-action. If $K$ is the triangulation of an $(n-1)$-dimensional sphere, the moment angle complex $Z_{K}$ is an $(m+n)$-dimensional manifold. The tangent bundle $\tau_{Z}$ is a $T^{m}$-equivariant real vector bundle. Applying the Borel construction establishes a vector bundle $\left(\tau_{Z}\right)_{h T^{m}}$ over $\left(Z_{K}\right)_{h T^{m}} \simeq D J(K)$ which is stably isomorphic to $\lambda_{\mathbb{R}}$. In particular, $\left(\tau_{Z}\right)_{h T^{m}}$ and $\xi_{\mathbb{R}}$ have equal Pontrjagin classes. Some details of the construction are recalled in the next section.

An oriented quasi-toric manifold $M^{2 n}$ is an oriented $2 n$-dimensional manifold with a $T^{n}$-action such that the action is locally standard and the orbit space $M^{2 n} / T^{n}=P$ is a simple polytope. We apply the Borel construction to the tangent bundle $\tau_{M}$ of $M^{2 n}$ and get a vector bundle $\left(\tau_{m}\right)_{h T^{n}}$ over $\left(M^{2 n}\right)_{h T^{n}} \simeq D J\left(K_{P}\right)$, where $K_{P}$ is the simplicial complex dual to the boundary of $P$. Again, $\left(\tau_{M}\right)_{h T^{n}}$ and $\lambda_{\mathbb{R}}$ are stably isomorphic, and $\left(\tau_{M}\right)_{h T^{n}}$ and $\xi_{\mathbb{R}}$ have the same Pontrjagin classes. All this can be found in DJ, Sections 4 and 6].

Theorem 1.2 allows us to draw the following corollary, which improves the stable isomorphism result about $\lambda$ and $\left(\tau_{M}\right)_{h T^{n}}$ [DJ, Theorem 6.6].

Corollary 1.6. (i) If $K$ is the triangulation of an $(n-1)$-dimensional sphere, then $\left(\tau_{Z}\right)_{h T^{m}}$ and $\xi_{\mathbb{R}} \oplus \mathbb{R}^{m-n}$ are isomorphic.

(ii) If $M^{2 n} \longrightarrow P$ is an oriented quasi-toric manifold over the simple polytope $P$, then $\left(\tau_{M}\right)_{h T^{n}} \oplus \mathbb{R}$ and $\xi_{\mathbb{R}} \oplus \mathbb{R}$ are isomorphic.

The connected sum $\mathbb{C} P(2) \sharp \mathbb{C} P(2)$ of $\mathbb{C} P(2)$ with itself is a quasi-toric manifold over a square, but does not admit an almost complex structure DJ, Section 0 (C') and Example 1.19], [BP page 68]. In particular, for $M=\mathbb{C} P(2) \sharp \mathbb{C} P(2)$, the real vector bundle $\left(\tau_{M}\right)_{h T^{n}}$ has no complex structure. This shows that in this case $\xi_{\mathbb{R}}$ and $\left(\tau_{M}\right)_{h T^{n}}$ cannot be isomorphic and that we cannot omit the trivial summand in the second part of the above corollary as well as in Theorem 1.2(ii).

Equivariant and non-equivariant almost complex structures for quasi-toric manifolds are discussed in [BP, Section 5]. Every equivariant complex structure for a quasi-toric manifold $M$ establishes a complex structure for the Borel construction $\left(\tau_{M}\right)_{h T^{n}}$. Since the total Pontrjagin class of $\left(\tau_{M}\right)_{h T^{n}}$ equals $\prod_{i=1}^{m}\left(1-v_{i}^{2}\right)$, Theorem 1.4 tells us that the Euler class $e\left(\left(\tau_{M}\right)_{h T^{n}}\right)$ decides whether $\left(\tau_{M}\right)_{h T^{n}}$ admits a complex structure. The calculation of $e\left(\left(\tau_{M}\right)_{h T^{n}}\right)$ can be worked out, but it will be discussed in a forthcoming paper DN]. It turns out that $\left(\tau_{M}\right)_{h T^{n}}$ has a complex structure if and only if $\tau_{M}$ has one. The combinatorial conditions for both cases are the same. For a few more details see Section 7 .

In a recent paper $[\mathrm{K}$, Kustarev gave a combinatorial condition, described in terms of omniorientations of the quasi-toric manifold $M$, which is sufficient and necessary for the existence of an equivariant almost complex structure for $M$. One can show that his condition and our condition established in Theorem 1.4 are equivalent [DN]. In contrast to his result we are able to give a complete classification of all complex structures for $\left(\tau_{M}\right)_{h T^{n}}$. For example, we have the following classification result. We say that two complex structures $\eta_{1}$ and $\eta_{2}$ for a real vector bundle $\rho$ are isomorphic if $\eta_{1} \cong \eta_{2}$ as complex vector bundles. 
Corollary 1.7. Let $K$ be an $(n-1)$-dimensional abstract finite simplicial complex and let $s \geq n$. Let $\rho$ be a $2 s$-dimensional oriented real vector bundle over $D J(K)$ such that $p(\rho)=p(K)$.

(i) If $s=n$, the number of isomorphism classes of complex structures for $\rho$ equals the number of functions $f:[m] \longrightarrow\{ \pm 1\}$ such that $e_{f}(K)= \pm e(\rho)$. In particular, there exists only a finite number of isomorphism classes of complex structures for $\rho$.

(ii) If $s>n$, then there exist exactly $2^{m}$ non-isomorphic complex structures for $\rho$.

In Section 7 we will give an explicit description of the complex structures of the bundle $\rho$. In fact, for each function $f:[m] \longrightarrow\{ \pm 1\}$, we will construct an $n$-dimensional complex vector bundle $\xi_{f}$ over $D J(K)$. The complex structures are represented by the bundles $\xi_{f}$ with $e_{f}(K)= \pm e(\rho)$, respectively, $\xi_{f} \oplus \mathbb{C}^{s-n}$.

Theorem 1.2 also allows us to relate colorings of simplicial complexes to stable splittings of the vector bundle $\xi$. A regular $r$-paint coloring, an $r$-coloring for short, of a simplicial complex $K$ is a non-degenerate simplicial map $K \longrightarrow \Delta[r]$, i.e. each face of $K$ is mapped isomorphically onto a face of $\Delta[r]$. If $\operatorname{dim} K=n-1$, then $K$ only allows $r$-colorings for $r \geq n$. The inclusion $K \subset \Delta([m])$ always provides an $m$-coloring.

Corollary 1.8. For an $(n-1)$-dimensional simplicial complex $K$ the following conditions are equivalent:

(i) $K$ admits an $r$-coloring $K \longrightarrow \Delta[r]$.

(ii) The vector bundle $\xi \oplus \mathbb{C}^{r-n} \cong \bigoplus \nu_{i}$ splits into a direct sum of complex line bundles.

(iii) The vector bundle $\xi_{\mathbb{R}} \oplus \mathbb{R}^{2(r-n)} \cong \bigoplus_{i=1}^{r} \theta_{i}$ splits into a direct sum of 2dimensional real vector bundles.

Proof. The proof is based on a similar result for colorings and splittings of $\lambda$ and $\lambda_{\mathbb{R}}$. We can assume that $n \leq r \leq m$. An $r$-coloring $K \longrightarrow \Delta([r])$ establishes a splitting $\lambda \cong \bigoplus_{i=1}^{r} \nu_{i} \oplus \mathbb{C}^{m-r}$ [N2]. The bundles $\bigoplus_{i=1}^{r} \nu_{i}$ and $\xi \oplus \mathbb{C}^{r-n}$ have the same Chern classes. Theorem 1.2 shows that both vector bundles are isomorphic and produces the desired splitting for $\xi \oplus \mathbb{C}^{r-n}$. The realification of both sides establishes a splitting for $\xi_{\mathbb{R}} \oplus \mathbb{R}^{2(m-r)}$. A splitting of $\xi_{\mathbb{R}} \oplus \mathbb{R}^{2(r-n)}$ produces a splitting for $\lambda_{\mathbb{R}} \cong \xi_{\mathbb{R}} \oplus \mathbb{R}^{2(m-n)}$. Also, such a splitting establishes a coloring $K \longrightarrow \Delta([r])$ [N2].

The paper is organized as follows. In the next section we describe different models for $D J(K)$ needed for our purposes. A geometric construction of the vector bundle $\xi$ with the properties stated in Theorem 1.1 is contained in Section 3 . Using Sullivan's arithmetic square we reduce the global uniqueness problem for our bundles to the analogous $p$-adic question in Section 4. This section also contains a proof of Theorem 1.3. The $p$-adic case involves the calculation of some higher derived inverse limits. In Section 5 we provide some general remarks about higher limits and filtrations of functors, needed in Section 6 for the proof of the $p$-adic existence and uniqueness statements. In the last section we discuss complex structures for real vector bundles and prove Theorem 1.4 and Corollary 1.7

If not specified otherwise, $K$ will always denote an abstract finite simplicial complex of dimension $n-1$ with $m$ vertices and $M_{K}$ the set of maximal faces. We also fix the classes $c(K)=\prod_{i=1}^{m}\left(1+v_{i}\right)$ and $p_{K}=\prod_{i=1}^{m}\left(1-v_{i}^{2}\right)$. 


\section{Models For $D J(K)$}

For the proof of our theorems we will need different models for the space $D J(K)$, which we will describe in this section.

Let $\operatorname{CAT}(K)$ be the category whose objects are the faces of $K$ and whose arrows are given by the subset relations between the faces. $\operatorname{CAT}(K)$ has an initial object given by the empty face. Given a pair $(X, Y)$ of pointed topological space we can define covariant functors

$$
X^{K},(X, Y)^{K} \text {. }
$$

The functor $X^{K}$ assigns to each face $\alpha$ the cartesian product $X^{\alpha}$ and to each morphism $i_{\alpha, \beta}$ the inclusion $X^{\alpha} \subset X^{\beta}$, where missing coordinates are set to the base point $*$. If $\alpha=\emptyset$, then $X^{\alpha}$ is a point. Also, $(X, Y)^{K}$ assigns to $\alpha$ the product $X^{\alpha} \times Y^{[m] \backslash \alpha}$ and to $i_{\alpha, \beta}$ the coordinate-wise inclusion $X^{\alpha} \times Y^{[m] \backslash \alpha} \subset X^{\beta} \times Y^{[m] \backslash \beta}$. The functors $X^{K}$ and $(X, Y)^{K}$ are called polyhedral product functors and are discussed in BBCG]. The inclusions $X^{\alpha} \subset X^{[m]}=X^{m}$ and $X^{\alpha} \times Y^{[m]-\alpha} \subset$ $X^{\alpha} \times X^{[m]-\alpha}=X^{m}$ establish inclusions

$$
\operatorname{colim}_{\mathrm{CAT}(K)} X^{K} \longrightarrow X^{m}, \operatorname{colim}_{\mathrm{CAT}(K)}(X, Y)^{K} \longrightarrow X^{m} .
$$

We are only interested in two particular cases, namely the functor $X^{K}$ for the classifying space $B T=\mathbb{C} P(\infty)$ of the 1-dimensional circle $T$ (considered as a topological group) and the functor $(X, Y)^{K}$ for the pair $\left(D^{2}, S^{1}\right)$. The colimit

$$
Z_{K}:=\operatorname{colim}_{\mathrm{CAT}(K)}\left(D^{2}, S^{1}\right)^{K}
$$

is called the moment angle complex associated to $K$. We have inclusions $Z_{K} \subset$ $\left(D^{2}\right)^{m} \subset \mathbb{C}^{m}$, and the standard $T^{m}$-action on $\mathbb{C}^{m}$ restricts to $Z_{K}$. The Borel construction produces a fibration

$$
q_{K}:\left(Z_{K}\right)_{h T^{m}} \longrightarrow B T^{m}
$$

with fiber $Z_{K}$. Moreover, $B_{T} K:=\left(Z_{K}\right)_{h T^{m}}$ is a realization of the Stanley-Reisner algebra $\mathbb{Z}[K]$. That is, there exists an isomorphism $H^{*}\left(B_{T} K ; \mathbb{Z}\right) \cong \mathbb{Z}[K]$ such that the map $H^{*}\left(q_{K} ; \mathbb{Z}\right)$ can be identified with the map $\mathbb{Z}[m] \longrightarrow \mathbb{Z}[K]$ [DJ, Theorem 4.8]. We will use this model for the geometric construction of our vector bundle $\xi$.

Buchstaber and Panov gave a different construction for $D J(K)$. They showed that the colimit $c(K):=\operatorname{colim}_{\mathrm{CAT}(K)} B T^{K}$ is homotopy equivalent to $B_{T} K$ and that the inclusion

$$
c(K) \longrightarrow B T^{m}
$$

is homotopic to $q_{K}[\mathrm{BP}$, Theorem 6.29].

We wish to study homotopy theoretic properties of $c(K)$. Colimits behave poorly from a homotopy theoretic point of view, but the left derived functor, known as the homotopy colimit, provides the appropriate tool for such questions. Following [V], the homotopy colimit $h c(K):=\operatorname{hocolim}_{\mathrm{CAT}(K)} B T^{K}$ may be described as the two-sided bar construction $B\left(*, \operatorname{CAT}(K), B T^{K}\right)$ in Top. For the functor $B T^{K}$, the map

$$
\operatorname{hocolim}_{\mathrm{CAT}(K)} B T^{K} \longrightarrow \operatorname{colim}_{\mathrm{CAT}(K)} B T^{K}
$$

is a homotopy equivalence [NR1. In particular, $h c(K) \simeq c(K)$. We will use the model $h c(K)$ to prove the uniqueness properties of the vector bundle $\xi$ and to provide a homotopy theoretic construction of $\xi$.

For later purposes we include the following remark. 
Remark 2.1. For $\alpha \subset[m]$ we denote by $\mathbb{Z}[\alpha]$ the polynomial algebra generated by elements $v_{i}, i \in \alpha$, of degree 2 , one for each vertex in $\alpha$. The projection $\mathbb{Z}[m] \longrightarrow \mathbb{Z}[\alpha]$ which maps $v_{i}$ to $v_{i}$ if $i \in \alpha$ and to 0 otherwise is induced by the inclusion $i_{\alpha,[m]}: B T^{\alpha} \longrightarrow B T^{m}$. By construction, this projection factors uniquely through a map $q_{\alpha}: \mathbb{Z}[K] \longrightarrow \mathbb{Z}[\alpha]$. Also, this map is induced by the inclusion $B T^{\alpha} \longrightarrow D J(K)$ which comes from the description of $D J(K)$ as a colimit or homotopy colimit. The direct sum of the maps $q_{\alpha}$ induces a monomorphism of algebras $\mathbb{Z}[K] \longrightarrow \bigoplus_{\alpha \in K} \mathbb{Z}[\alpha]$.

\section{Geometric construction of vector bundles}

The $m$-dimensional torus $T^{m}$ acts on $\mathbb{C}^{m}$ via coordinate-wise multiplication. Let $\lambda^{\prime} \downarrow Z_{K}$ denote the $m$-dimensional $T^{m}$-equivariant complex vector bundle over $Z_{K}$ given by the diagonal action of $T^{m}$ on $\mathbb{C}^{m} \times Z_{K}$. The Borel construction produces an $m$-dimensional complex bundle $\lambda:=\lambda_{h T^{m}}^{\prime}$ over $D J(K)$ which is isomorphic to the pull back of the universal bundle over $B U(m)$ along the composition of $q_{K}: D J(K) \longrightarrow B T^{m}$ and the maximal torus inclusion $B T^{m} \longrightarrow B U(m)$. As already mentioned, we have $c(\lambda)=c(K)$ (for details see [DJ, Section 6]).

For $x, z \in \mathbb{C}^{m}$ we denote by $\bar{z}$ the coordinate-wise complex conjugate of $z$ and by $x z \in \mathbb{C}^{m}$ the coordinate-wise product of $x$ and $z$; that is, that the $i$-th coordinate $(x z)_{i}$ of $x z$ is given by the product $x_{i} z_{i}$. Let $A \in \mathbb{C}^{(m-n) \times m}$ be an $[(m-n) \times m]$ matrix. Each face $\alpha \in K$ defines a submatrix $A_{\alpha} \in \mathbb{C}^{(m-n) \times(m-|\alpha|)}$ given by the columns indexed by the elements not in $\alpha$. The matrix $A$ is called $K$-admissible if for each face $\alpha \in K$ the matrix $A_{\alpha}$ is an epimorphism. For example, the matrix $A=\left(s^{r}\right), 1 \leq r \leq m, 1 \leq s \leq m-n$, is $K$-admissible for any $(n-1)$-dimensional complex with $m$-vertices.

We define a map

$$
f_{A}: \mathbb{C}^{m} \times Z_{K} \longrightarrow \mathbb{C}^{m-n} \times Z_{K}
$$

by $f_{A}(x, z):=(y, z)$, where $y:=A(x \bar{z})$. Here, we use that $Z_{K} \subset \mathbb{C}^{m}$. If $T^{m}$ acts on the target only via the second coordinate, then the space $E(m-n):=\mathbb{C}^{m-n} \times Z_{K}$ is the total space of the $(m-n)$-dimensional trivial $T^{m}$-bundle $\mathbb{C}^{m-n} \downarrow Z_{K}$. The source is the total space $E\left(\lambda^{\prime}\right)$ of the $T^{m}$-bundle $\lambda^{\prime}$.

Proposition 3.1. Let $K$ be an $(n-1)$-dimensional complex and $A \in \mathbb{C}^{(m-n) \times m}$ be a $K$-admissible matrix.

(i) The map $f_{A}: E\left(\lambda^{\prime}\right) \longrightarrow E(m-n)$ is $T^{m}$-equivariant and a bundle epimorphism.

(ii) There exists a $T^{m}$-equivariant $n$-dimensional complex vector bundle $\xi^{\prime} \downarrow Z_{K}$ such that $\lambda^{\prime} \cong \mathbb{C}^{m-n} \oplus \xi^{\prime}$ as $T^{m}$-equivariant vector bundles over $Z_{K}$.

(iii) We have $\lambda \cong \mathbb{C}^{m-n} \oplus \xi_{h T^{m}}^{\prime}$ as vector bundles over $D J(K)$.

(iv) The total Chern class of $\xi_{h T^{m}}^{\prime}$ is given by $c\left(\xi_{h T^{m}}^{\prime}\right)=c(K) \in \mathbb{Z}[K]$.

(v) The total Pontrjagin class of $\left(\xi_{h T^{m}}^{\prime}\right)_{\mathbb{R}}$ is given by $p\left(\left(\xi_{h T^{m}}^{\prime}\right)_{\mathbb{R}}\right)=p(K) \in \mathbb{Z}[K]$.

Proof. For $t \in T^{m}$, we have $f_{A}(t(x, z))=f_{A}(t x, t z)=(A(t x \bar{t} \bar{z}), t z)=(A(x \bar{z}), t z)=$ $t f_{A}(x, z)$. This shows that $f_{A}$ is $T^{m}$-equivariant. Every point $z=\left(z_{1}, \ldots, z_{m}\right) \in Z_{K}$ is contained in $\left(S^{1}\right)^{[m]-\alpha} \times\left(D^{2}\right)^{\alpha}$ for some maximal face $\alpha \in K$. In particular, $z_{i} \neq 0$ for $i \notin \alpha$. Since the matrix $A_{\alpha}$ is invertible, this shows that the restriction of $f_{A}$ to the fiber over $z$ is an epimorphism and that $f_{A}$ is a bundle epimorphism. This proves the first part. 
The kernel $\xi^{\prime}:=\operatorname{ker} f_{A}$ is again a $T^{m}$-equivariant vector bundle over $Z_{K}$. Since for a compact Lie group $G$ every short exact sequence of $G$-equivariant vector bundles over compact spaces splits [S], we get $\lambda^{\prime} \cong \xi^{\prime} \oplus \mathbb{C}^{m-n}$. This proves the second part. The other three parts are direct consequences of part (ii).

Remark 3.2. This gives a geometric construction of the vector bundle $\xi:=\xi_{h T^{m}}^{\prime}$, whose existence was claimed in Theorem 1.1. We will give an alternative proof of Theorem 1.1 in the later sections.

The construction of $\xi$ depends on the chosen matrix A. But, as Theorem 1.2 says, the isomorphism type is independent of $A$.

\section{UNIQUENESS PROPERTIES OF $\xi$}

In this section we describe vector bundles by their classifying maps. That is, an $r$-dimensional complex vector bundle $\eta$ over a space $X$ is a map $\eta: X \longrightarrow B U(r)$. Then, Theorem 1.1 and Theorem 1.2 become statements about the existence of maps and the number of homotopy classes of maps realizing prescribed cohomological data given by characteristic classes. As usual, such problems are best approached by considering separately their rational and $p$-adic versions. An application of Sullivan's arithmetic square will then provide the desired global information. As a side effect we will also provide a homotopy theoretic construction of the vector bundle $\xi: D J(K) \longrightarrow B U(n)$, already constructed in Section 3 .

Let $U(r) \longrightarrow S O(2 r) \longrightarrow S O(2 r+1)$ denote the standard inclusions of the compact Lie groups $U(r), S O(2 r)$ and $S O(2 r+1)$. Composition with $B U(r) \longrightarrow$ $B S O(2 r)$ induces the realification of a complex vector bundle $\eta: X \longrightarrow B U(r)$ and, as in the introduction, will be denoted by $\eta_{\mathbb{R}}$. Composition with $B S O(2 r) \longrightarrow$ $B S O(2 r+1)$ means that we add a 1-dimensional trivial bundle. If we think of $T^{r}$ as the set of diagonal matrices in $U(r)$, the compositions

$$
T^{r} \longrightarrow U(r) \longrightarrow S O(2 r) \longrightarrow S O(2 r+1)
$$

describe at each stage the standard maximal torus of the compact Lie group. The integral cohomology $H^{*}\left(B T^{r} ; \mathbb{Z}\right) \cong \mathbb{Z}\left[v_{1}, \ldots, v_{r}\right]=\mathbb{Z}[r]$ is a polynomial algebra with $r$ generators in degree 2. Passing to classifying spaces and cohomology establishes maps

$$
\begin{aligned}
& H^{*}(B U(r) ; \mathbb{Z}) \longrightarrow \mathbb{Z}[r]^{W_{U(r)}}=\mathbb{Z}\left[c_{1}, \ldots, c_{r}\right] \subset \mathbb{Z}[r], \\
& H^{*}(B S O(2 r) ; \mathbb{Z}) \longrightarrow \mathbb{Z}[r]^{W_{S O}(2 r)}=\mathbb{Z}\left[p_{1}, \ldots, p_{r-1}, e_{r}\right] \subset \mathbb{Z}[r], \\
& H^{*}(B S O(2 r+1) ; \mathbb{Z}) \longrightarrow \mathbb{Z}[r]^{W_{S O}(2 r+1)}=\mathbb{Z}\left[p_{1}, \ldots, p_{r}\right] \subset \mathbb{Z}[r],
\end{aligned}
$$

where $W_{U(r)}, W_{S O(2 r)}$ and $W_{S O(2 r+1)}$ denote the Weyl groups, $c_{i}$ the universal Chern classes for complex, $p_{i}$ the universal Pontrjagin classes for real and $e_{r}$ the universal Euler class for oriented $2 r$-dimensional real vector bundles. The class $c_{i}$ is given by the $i$-th elementary symmetric polynomial $\sigma_{i}\left(v_{1}, \ldots, v_{r}\right)$, the class $e_{r}$ by $\sigma_{r}\left(v_{1}, \ldots, v_{r}\right)$ and $p_{i}$ by $\sigma_{i}\left(-v_{1}^{2}, \ldots,-v_{r}^{2}\right)$. The Euler class satisfies the equation $e_{r}^{2}=$ $(-1)^{r} p_{r}$. In all cases the arrow is an epimorphism, for $U(r)$ even an isomorphism. If we pass to rational coefficients all arrows become isomorphisms.

If $K$ is a finite simplicial complex of dimension $n-1$, the composition $\mathbb{Z}\left[c_{1}, \ldots, c_{m}\right]$ $\longrightarrow \mathbb{Z}[m] \longrightarrow \mathbb{Z}[K]$ maps the Chern class $c_{j}$ to zero for $j \geq n+1$. Hence this composition factors uniquely through $\mathbb{Z}\left[c_{1}, \ldots, c_{n}\right]$ and establishes a map $f: \mathbb{Z}\left[c_{1}, \ldots, c_{n}\right] \longrightarrow$ 
$\mathbb{Z}[K]$ and a commutative diagram

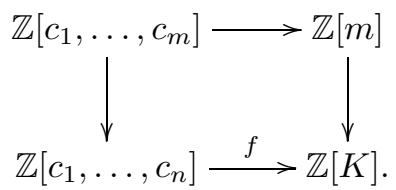

This diagram can partly be realized by spaces and maps, namely by

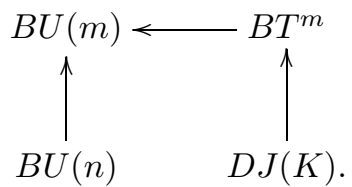

We want to construct a map $D J(K) \longrightarrow B U(n)$ making the above diagram commutative up to homotopy. Such a map is a realization of $f$ and establishes an $n$-dimensional complex vector bundle over $D J(K)$ which has the desired Chern classes. In fact, the bundle $\xi \downarrow D J(K)$ constructed in Section 3 is such a map. But here we will give another construction. We also want to show that such a map is unique up to homotopy.

We can play a similar game with $B S O(2 m)$ and $B S O(2 n)$. In this case, the composition $\mathbb{Z}\left[p_{1}, \ldots, p_{m-1}, e_{m}\right] \longrightarrow \mathbb{Z}[m] \longrightarrow \mathbb{Z}[K]$ factors through $\mathbb{Z}\left[p_{1}, \ldots, p_{n-1}, e_{n}\right]$ $\longrightarrow \mathbb{Z}[K]$. We have some freedom for the choice of the image of the Euler class $e_{n}$. Since $p_{n}$ is mapped to the non-trivial class $p_{n}(K):=(-1)^{n} \sigma_{n}\left(v_{1}^{2}, \ldots, v_{m}^{2}\right)$, each square root of $(-1)^{n} p_{n}(K)$ establishes a different factorization $\mathbb{Z}\left[p_{1}, \ldots, p_{n-1}, e_{n}\right] \longrightarrow \mathbb{Z}[K]$. Again, all these factorizations can be realized and are unique up to homotopy. Before we make these statements precise, we need a description of all square roots. The Euler classes $e_{\omega}$ associated to functions $\omega: M_{K} \longrightarrow\{ \pm 1\}$ and defined in the introduction provide a complete list of such square roots.

Lemma 4.1. Let $K$ be an $(n-1)$-dimensional simplicial complex. Then, every square root of $(-1)^{n} p_{n}(K)$ is of the form $e_{\omega}$.

Proof. Every square root $e$ of $(-1)^{n} p_{n}(K)=\sigma_{n}\left(v_{1}^{2}, \ldots, v_{m}^{2}\right)=\sum_{\mu \in M_{K}} v_{\mu}^{2}$ can be written as a linear combination $e=\sum_{i=1}^{r} a_{i} q_{i}$ of monomials $q_{i}$ which are of the form $\prod_{j=1}^{m} v_{j}^{l_{j}}$ with $l_{j} \geq 0$ and $\sum_{j} l_{j}=n$. We can compare the square $e^{2}=$ $\sum_{i} a_{i}^{2} q_{i}^{2}+\sum_{i<k} 2 a_{i} a_{j} q_{i} q_{k}$ with $\sum_{\mu \in M_{K}} v_{\mu}^{2}$. By Remark 2.1, both expressions are equal in $\mathbb{Z}[K]$ precisely when all non-trivial monomials have equal coefficients and $q_{i}^{2}=0$ if and only if $q_{i}=0$. Hence, if $\mu \in K$ is a maximal face, the coefficient of $v_{\mu}$ in $e$ is \pm 1 and all other coefficients vanish. This shows that $e=e_{\omega}$ for an appropriate function $\omega: M_{K} \longrightarrow\{ \pm 1\}$.

As already explained each class $e_{\omega}$ establishes a unique factorization

$$
h_{\omega}: \mathbb{Z}\left[p_{1}, \ldots, p_{n-1}, e_{n}\right] \longrightarrow \mathbb{Z}[K] .
$$

We say that a map $\rho_{\omega}: D J(K) \longrightarrow B S O(2 n)$ realizes $h_{\omega}$ if $H^{*}\left(\rho_{\omega} ; \mathbb{Q}\right)=h_{\omega} \otimes \mathbb{Q}$.

Theorem 4.2. Let $K$ be an $(n-1)$-dimensional finite simplicial complex and $\omega: M_{K} \longrightarrow\{ \pm 1\}$ a function. Then there exist realizations

$$
\xi: D J(K) \longrightarrow B U(n) \text { and } \rho_{\omega}: D J(K) \longrightarrow B S O(2 n)
$$

of $f \otimes \mathbb{Q}$ and $h_{\omega} \otimes \mathbb{Q}$. 
The uniqueness question can be handled simultaneously for the different cases. For $n \leq r$ we denote by $G_{r}$ one of the groups $U(r), S O(2 r)$ or $S O(2 r+1)$ and by $\gamma_{r}: D J(K) \longrightarrow B G_{r}$ one of the maps $\xi \oplus \mathbb{C}^{r-n}, \rho_{\omega} \oplus \mathbb{R}^{2(n-r)}$ or $\xi_{\mathbb{R}} \oplus \mathbb{R}^{2(r-n)+1}$.

Theorem 4.3. Let $K$ be an $(n-1)$-dimensional finite simplicial complex. Then, a map $\beta_{r}: D J(K) \longrightarrow B G_{r}$ is homotopic to $\gamma_{r}$ if and only if $H^{*}\left(\beta_{r} ; \mathbb{Q}\right)=H^{*}\left(\gamma_{r} ; \mathbb{Q}\right)$.

The above two results are versions of our first two main theorems.

Proof of Theorem 1.1 and Theorem 1.2. Theorem 1.1 is contained in Theorem 4.2 and Theorem 1.2 (i) and (iii) in Theorem 4.3. The non-vanishing of $c_{n}(\xi)$ and $p_{n}\left(\xi_{\mathbb{R}}\right)$ follows from the fact that $K$ contains a face of dimension $n-1$. The inequalities $r \geq n$ and $s \geq 2 n$ follow from the non-vanishing of these Chern and Pontrjagin classes. Since $D J(K)$ is simply connected, every real vector bundle $\rho \downarrow D J(K)$ can be given an orientation. If $\operatorname{dim} \rho>2 n$, then $e(\rho)=0=e\left(\xi_{\mathbb{R}} \oplus \mathbb{R}^{s-2 n}\right)$ and if $\operatorname{dim} \rho=2 n$, then the same formula holds for $\rho \oplus \mathbb{R}$ and $\xi_{\mathbb{R}} \oplus R$. In both cases, Theorem 4.3 establishes the desired isomorphism between the vector bundles.

As already mentioned the proofs of the above theorems are based on an arithmetic square argument and rational and $p$-adic versions of the above statements. In the rest of this section we will reduce the proof of Theorem 4.2 and Theorem 4.3 to their $p$-adic versions.

For a topological space $X$ we denote by $X_{0}$ the rationalization, by $X_{p}^{\wedge}$ the $p$-adic completion in the sense of Bousfield and Kan, by $X^{\wedge}:=\prod_{p} X_{p}^{\wedge}$ the product of all $p$-adic completions, and by $X_{\mathbb{A}_{f}}$ the finite adele type of $X$, which is the formal completion of $X_{0}$ or the rationalization of $X^{\wedge}$. All spaces under consideration are simply connected. In this case Sullivan's arithmetic square

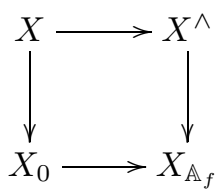

is a homotopy pull back diagram.

Theorem 4.4. Let $K$ be an $(n-1)$-dimensional finite simplicial complex and $\omega: M_{K} \longrightarrow\{ \pm 1\}$ a function. Then there exist realizations

$$
\xi_{0}: D J(K) \longrightarrow B U(n)_{0} \text { and }\left(\rho_{\omega}\right)_{0}: D J(K) \longrightarrow B S O(2 n)_{0}
$$

of $f \otimes \mathbb{Q}$ and $h_{\omega} \otimes \mathbb{Q}$.

Theorem 4.5. Let $K$ be an $(n-1)$-dimensional finite simplicial complex. A map $\left(\beta_{r}\right)_{0}: D J(K) \longrightarrow\left(B G_{r}\right)_{0}$ is homotopic to $\left(\gamma_{r}\right)_{0}$ if and only if $H^{*}\left(\left(\beta_{r}\right)_{0} ; \mathbb{Q}\right)=$ $H^{*}\left(\gamma_{r} ; \mathbb{Q}\right)$.

Proof of both theorems. All claims follow from the fact that $\left(B G_{r}\right)_{0}$ is a product of rational Eilenberg-Mac Lane spaces.

For abbreviation, we define $H_{\mathbb{Q}_{\hat{p}}^{\wedge}}^{*}(-):=H^{*}\left(-; \mathbb{Z}_{p}^{\wedge}\right) \otimes_{\mathbb{Z}_{p}^{\wedge}} \mathbb{Q}_{p}^{\wedge}$.

Theorem 4.6. Let $p$ be a prime, $K$ an $(n-1)$-dimensional finite simplicial complex, and $\omega: M_{K} \longrightarrow\{ \pm 1\}$ a function. Then there exist realizations

$$
\xi_{p}^{\wedge}: D J(K) \longrightarrow B U(n)_{p}^{\wedge} \text { and }\left(\rho_{\omega}\right)_{p}^{\wedge}: D J(K) \longrightarrow B S O(2 n)_{p}^{\wedge}
$$

of $f \otimes_{\mathbb{Z}} \mathbb{Q}_{p}^{\wedge}$ and $h_{\omega} \otimes_{\mathbb{Z}} \mathbb{Q}_{p}^{\wedge}$. 
Theorem 4.7. Let $p$ be a prime and $K$ an $(n-1)$-dimensional finite simplicial complex. A map $\left(\delta_{r}\right)_{p}^{\wedge}: D J(K) \longrightarrow\left(B G_{r}\right)_{p}^{\wedge}$ is homotopic to the completion $\left(\gamma_{r}\right)_{p}^{\wedge}$ of $\gamma_{r}$ if and only if $H_{\mathbb{Q}_{p}^{\wedge}}^{*}\left(\left(\delta_{r}\right)_{p}^{\wedge}\right)=H_{\mathbb{Q}_{p}^{\wedge}}^{*}\left(\left(\gamma_{r}\right)_{p}^{\wedge}\right)$.

The proof of the last two theorems will be postponed to Section 6 .

The following statement is necessary for the proof of Theorem 4.3 ,

Theorem 4.8. The map

$$
\left[D J(K), B G_{r}\right] \longrightarrow\left[D J(K), B G_{r}^{\wedge}\right]
$$

is a monomorphism.

Proof. Since the arithmetic square for $B G_{r}$ is a pull back, the homotopy fiber $F$ of $B G_{r} \longrightarrow B G_{r}^{\wedge}$ is equivalent to the homotopy fiber of the rationalization $\left(B G_{r}\right)_{0} \longrightarrow$ $\left(B G_{r}\right)_{\mathbb{A}_{f}}$. Since $\left(B G_{r}\right)_{0}$ is a product of rational Eilenberg-Mac Lane spaces of even degrees, $\pi_{i}(F)=0$ for $i$ even. The obstruction groups for lifting homotopies between maps $D J(K) \longrightarrow B G_{r}^{\wedge}$ to $B G_{r}$ are given by $H^{*}\left(D J(K) ; \pi_{*}(F)\right)$. All these obstruction groups vanish, since $H^{*}(D J(K) ; \mathbb{Z})$ is concentrated in even degrees.

Proof of Theorem 4.2. Since the rationalization $B U(n)_{0}$ is a product of rational Eilenberg-Mac Lane spaces of even degrees, the same holds for the finite adele type $B U(n)_{\mathbb{A}_{f}}$. Up to homotopy, maps into $B U(n)_{\mathbb{A}_{f}}$ are determined by cohomological information. Since $\xi_{0}$ and $\xi_{p}^{\wedge}$ realize $f \otimes \mathbb{Q}$, respectively, $f \otimes \mathbb{Q}_{p}^{\wedge}$, the two compositions

$$
D J(K) \stackrel{\xi_{0}}{\longrightarrow} B U(n)_{0} \longrightarrow B U(n)_{\mathbb{A}_{f}}
$$

and

$$
D J(K) \stackrel{\prod \xi_{p}^{\wedge}}{\longrightarrow} \prod_{p} B U(n)_{p}^{\wedge}=B U(n)^{\wedge} \longrightarrow B U(n)_{\mathbb{A}_{f}}
$$

are homotopic. Using the homotopy pull back property of the arithmetic square for $B U(n)$ we can construct a map $\xi: D J(K) \longrightarrow B U(n)$ with the desired cohomological property. This proves the claim for $B U(n)$. For $B S O(2 n)$ we can argue analogously.

Proof of Theorem 4.3 . Since $H^{*}(D J(K) ; \mathbb{Z})$ is torsion free, the map $H^{*}\left(\gamma_{r} ; \mathbb{Q}\right)$ determines $H^{*}\left(\gamma_{r} ; \mathbb{Z}\right)$ as well as $H^{*}\left(\rho ; \mathbb{Z}_{p}^{\wedge}\right) \otimes \mathbb{Q}_{p}^{\wedge}$. The homotopical uniqueness of $\gamma_{r}$ follows from Theorem 4.7 and Theorem 4.8 .

Proof of Theorem 1.3. If the total Pontrjagin class $p(\rho)$ of an oriented vector bundle $\rho: D J(K) \longrightarrow B S O(2 n)$ equals $p(K)$, then $e(\rho)^{2}=(-1)^{n} p_{n}(K)$. By Lemma 4.1 there exists a function $\omega: M_{K} \longrightarrow\{ \pm 1\}$ such that $e(\rho)=e_{\omega}$ and by Theorem 1.2 this implies that $\rho$ and $\rho_{\omega}$ are isomorphic.

\section{Higher Limits}

The proofs of Theorems 4.6 and 4.7 involve the calculation of some higher derived limits for covariant functors defined on the opposite category CAT $(K)^{o p}$ of $\operatorname{CAT}(K)$. For a definition and properties of higher derived limits see $[\mathrm{BK}]$ or $\mathrm{O}$. We will drop $\operatorname{CAT}(K)^{o p}$ from the notation of limits. 
Let $\mathrm{AB}$ denote the category of abelian groups. We would like to construct a filtration for a functor $\Phi: \operatorname{CAT}(K)^{o p} \longrightarrow \mathrm{AB}$. For $0 \leq s \leq n$ we define functors $\Phi_{\leq s}, \Phi_{s}: \operatorname{CAT}(K)^{o p} \longrightarrow \mathrm{AB}$ by

$$
\Phi_{\leq s}(\alpha):=\left\{\begin{array}{ll}
\Phi(\alpha) & \text { for }|\alpha| \leq s, \\
0 & \text { for }|\alpha|>s,
\end{array} \quad \Phi_{s}(\alpha):= \begin{cases}\Phi(\alpha) & \text { for }|\alpha|=s \\
0 & \text { for }|\alpha| \neq s\end{cases}\right.
$$

Since for $|\alpha|<|\beta|$ there is no arrow $\alpha \rightarrow \beta$ in $\operatorname{CAT}(K)^{o p}$, both functors are well defined. Moreover, we have $\Phi_{\leq n}=\Phi$. There exist short exact sequences of functors

$$
1 \longrightarrow \Phi_{\leq s-1} \longrightarrow \Phi_{\leq s} \longrightarrow \Phi_{s} \longrightarrow 1
$$

which induce long exact sequences

$$
\ldots \rightarrow \lim ^{i-1} \Phi_{s} \rightarrow \lim ^{i} \Phi_{\leq s-1} \rightarrow \lim ^{i} \Phi_{\leq s} \rightarrow \lim ^{i} \Phi_{s} \longrightarrow \ldots
$$

of higher derived limits.

\section{Lemma 5.1.}

(i) $\lim ^{i} \Phi_{s}=0$ for $i \geq n-s+1$.

(ii) $\lim ^{i} \Phi_{\leq s} \longrightarrow \lim ^{i} \Phi$ is an isomorphism for $s \geq n-i+1$.

(iii) If $i \geq \overline{1}$ and if $\Phi(\beta) \cong \Phi(\alpha)$ for $\alpha \subset \beta$ and $|\beta| \leq n-i+1$, then $\lim ^{i} \Phi=0$.

Proof. For each $s$ the functor $\Phi_{s} \cong \prod_{\alpha \in K,|\alpha|=s} \Phi_{\alpha}$ is a product of atomic functors $\Phi_{\alpha}$, i.e. $\Phi_{\alpha}(\beta)=0$ if $\beta \neq \alpha$. In [NR2] the higher limits of atomic functors are calculated. We have

$$
\lim ^{i} \Phi_{\alpha}=\widetilde{H}^{i-1}\left(\ell_{K}(\alpha) ; \Phi(\alpha)\right)
$$

where $\ell_{K}(\alpha):=\{\beta \in K: \alpha \cap \beta=\emptyset, \alpha \cup \beta \in K\}$ denotes the link of the face $\alpha$ in $K$. In particular, $\operatorname{dim}\left(\ell_{K}(\alpha)\right) \leq n-|\alpha|-1$. Hence, these groups vanish for $i \geq n-|\alpha|+1$. This proves the first part.

Since $\lim ^{j} \Phi_{s+1}=0$ for $j \geq n-s$, the second part follows from the above long exact sequences for higher derived limits.

Let $M:=\Phi(\emptyset)$ and let $\operatorname{cst}_{M}: \operatorname{CAT}(K)^{o p} \longrightarrow \mathrm{AB}$ denote the constant functor with value $M$; i.e. every face is mapped to $M$ and every morphism to the identity. Then, $\lim ^{i} \operatorname{cst}_{M}=0$ for $i \geq 1$ since $\operatorname{CAT}(K)^{o p}$ has a terminal object and is contractible BK]. By parts (i) and (ii), we get for $i \geq 1$

$$
\begin{aligned}
0=\lim ^{i} \operatorname{cst}_{M} \cong \lim ^{i}\left(\operatorname{cst}_{M}\right)_{\leq n-i+1} \\
\cong \lim ^{i} \Phi_{\leq n-i+1} \cong \lim ^{i} \Phi,
\end{aligned}
$$

which proves the third part.

\section{THE $p$-ADIC CASE}

This section is devoted to the proof of Theorem 4.6 and Theorem 4.7. For the proof of Theorem 4.7 and for the next section we will need the following statement. Both parts may be found in N1.

Theorem 6.1. Let $G$ be a connected compact Lie group with maximal torus $T_{G} \subset G$ and let $S$ be a torus.

(i) The map $\operatorname{hom}\left(S, T_{G}\right) / W_{G} \longrightarrow[B S, B G]$ is a bijection.

(ii) The map $[B S, B G] \longrightarrow \operatorname{Hom}\left(H^{*}(B G ; \mathbb{Q}), H^{*}(B S ; \mathbb{Q})\right)$ is an injection. 
Remark 6.2. In fact, for the proof of Theorem 4.7 the $p$-adic version of the second part is necessary. That is, that

$$
\left[B S, B G_{p}^{\wedge}\right] \longrightarrow \operatorname{Hom}\left(H_{\mathbb{Q}_{p}^{\wedge}}^{*}(B G), H_{\mathbb{Q}_{p}^{\wedge}}^{*}(B S)\right)
$$

is a monomorphism. A similar argument as in the global case will work.

Since $B T_{G_{p}} \wedge$ is an Eilenberg-Mac Lane space with torsion free $p$-adic cohomology, the statement holds if we replace $B G$ by $B T_{G}$. Moreover, up to homotopy, every map $B S \longrightarrow B G_{p}^{\wedge}$ factors through the map $B T_{G_{p}} \longrightarrow B G_{p}^{\wedge}$ [DW, Proposition 8.11]. Factorizations $B S \longrightarrow B T_{G} \hat{p}$ of a map $\phi: B S \longrightarrow B G_{p}^{\wedge}$ define extensions $H_{\mathbb{Q}_{\hat{p}}^{\wedge}}^{*}\left(B T_{G}\right) \longrightarrow H_{\mathbb{Q}_{\hat{p}}^{\wedge}}^{*}(B S)$ of $H_{\mathbb{Q}_{\hat{p}}^{\wedge}}^{*}(\phi): H_{\mathbb{Q}_{\hat{p}}^{\wedge}}^{*}(B G) \longrightarrow H_{\mathbb{Q}_{\hat{p}}^{\wedge}}^{*}(B S)$. But two extensions differ only by an element of the Weyl group $W_{G}$ of $G$. This is proved in AM, Theorem 1.7] for rational cohomology, but since $H_{\mathbb{Q}_{p}^{\wedge}}^{*}(B G) \cong H_{\mathbb{Q}_{p}^{\wedge}}^{*}\left(B T_{G}\right)^{W_{G}}$ and since $\mathbb{Q}_{p}^{\wedge}$ is an infinite field, the same argument works in our case.

Now we can argue as follows. Let $\phi, \psi: B T^{s} \longrightarrow B G_{p}^{\wedge}$ be two maps which induce the same map in $H_{\mathbb{Q}_{\hat{p}}^{\wedge}}^{*}(-)$-cohomology. We choose lifts $\hat{\phi}, \hat{\psi}: B S \longrightarrow B T_{G_{p}}$. By the above arguments there exists $w \in W_{G}$ such that $\hat{\phi} \simeq w \hat{\psi}$. Since conjugation by elements induces self maps of $B G$ homotopic to the identity, this shows that $\phi$ and $\psi$ are homotopic.

We will work with the model $h c(K)=\operatorname{hocolim}_{\mathrm{CAT}(K)} B T^{K}$ for $D J(K)$. Again all colimits, limits and higher derived limits are taken over $\operatorname{CAT}(K)$ or the opposite category $\operatorname{CAT}(K)^{o p}$. For simplification we will continue to drop these categories from the notation of limits. We will also skip the notation $\hat{p}$ from completions of maps. Again, $G_{r}$ will denote either $U(r), S O(2 r)$ or $S O(2 r+1)$, and $T^{r} \subset G_{r}$ the standard maximal torus. Again, $\operatorname{dim} K+1=n \leq r$.

The two theorems state the existence and uniqueness of a map $h c(K) \longrightarrow B G_{r_{p}}$, which realize some cohomological data, namely a particular map $g_{r}: H_{\mathbb{Q}_{p}^{\wedge}}^{*}\left(B G_{r}\right) \longrightarrow$ $H_{\mathbb{Q}_{p}^{\wedge}}^{*}(h c(K))$. The map $g_{r}$ will be $f \otimes_{\mathbb{Z}} \mathbb{Q}_{p}^{\wedge}, h_{\omega} \otimes_{\mathbb{Z}} \mathbb{Q}_{p}^{\wedge}$ or compositions with $H_{\mathbb{Q}_{p}^{\wedge}}^{*}(B U(r)) \longrightarrow H_{\mathbb{Q}_{p}^{\wedge}}^{*}(B U(n))$ or $H_{\mathbb{Q}_{p}^{\wedge}}^{*}(B S O(t)) \longrightarrow H_{\mathbb{Q}_{p}^{\wedge}}^{*}(B S O(2 n)), t \geq 2 n+1$.

For questions of this type we can use a well-known approach which in our case can be described by four different steps. In the following, $[-,-]$ denotes the set of homotopy classes of maps.

Step 1. For each piece of the decomposition $D J(K) \simeq h c(K)$, that is, for each $\alpha \in K$, we construct a map $\gamma_{\alpha}: B T^{\alpha} \longrightarrow B G_{r p} \hat{p}$ realizing the composition

$$
H_{\mathbb{Q}_{\hat{p}}^{\hat{p}}}^{*}\left(B G_{r}\right) \stackrel{g_{r}}{\longrightarrow} H_{\mathbb{Q}_{\hat{p}}^{\hat{n}}}^{*}(h c(K)) \stackrel{q_{\alpha}}{\longrightarrow} H_{\mathbb{Q}_{\hat{p}}^{\hat{n}}}^{*}\left(B T^{\alpha}\right)
$$

of $q_{\alpha}$, defined in Remark 2.1, and $g_{r}$.

Step 2. We need to show that, up to homotopy, the maps $\gamma_{\alpha}$ are compatible with the maps $B T^{\alpha} \longrightarrow B T^{\beta}$ induced by the arrows of the category $\operatorname{CAT}(K)$. That is, they define an element $\hat{\gamma}_{r}$ in the inverse limit $\lim \left[B T^{K}, B G_{r p} \wedge\right.$.

Since

$$
\begin{aligned}
\operatorname{map}\left(h c(K), B G_{r_{p}}\right) & \simeq \operatorname{map}\left(\operatorname{hocolim} B T^{K}, B G_{r_{p}}\right) \\
& \simeq \operatorname{holim} \operatorname{map}\left(B T^{K}, B G_{r p}\right),
\end{aligned}
$$

passing to path components and composing with

$$
\pi_{0}\left(\operatorname{holim} \operatorname{map}\left(B T^{K}, B G_{r p}^{\wedge}\right)\right) \longrightarrow \lim \left[B T^{K}, B G_{r p}^{\wedge}\right]
$$


defines a map

$$
R:\left[h c(K), B G_{r p}^{\wedge}\right] \longrightarrow \lim \left[B T^{K}, B G_{r p}^{\wedge}\right]
$$

We would like to know whether $R^{-1}\left(\hat{\gamma}_{r}\right)$ is non-empty, that is, whether there exists a map $\gamma_{r}: h c(K) \longrightarrow B G_{r_{p}}$ such that $\left.\gamma_{r}\right|_{B T^{\alpha}} \simeq \gamma_{\alpha}$ for all $\alpha \in K$. If this is the case, then we also would like to know how many elements are contained in $R^{-1}(\phi)$, that is, how many different homotopy classes of maps satisfy this property.

The Bousfield-Kan spectral sequence for homotopy inverse limits BK], together with work by Wojtkowiak [W] clarifying the situation in small degrees, provides an obstruction theory which may answer both questions. The obstruction groups are given by the higher derived limits of the functors

$$
\Pi_{i}^{G_{r}}: \mathrm{CAT}(K)^{o p} \longrightarrow \mathrm{AB}
$$

which are defined by $\Pi_{i}^{G_{r}}(\alpha):=\pi_{i}\left(\operatorname{map}\left(B T^{\alpha}, B G_{r p}^{\wedge}\right)_{\gamma_{\alpha}}\right)$.

If $\lim ^{i+1} \Pi_{i}^{G_{r}}=0$ for all $i \geq 1$, then $R^{-1}\left(\hat{\gamma}_{r}\right)$ is non-empty, and if in addition $\lim ^{i} \Pi_{i}^{G_{r}}=0$ for all $i \geq 1$, then the conditions $\left.\gamma_{r}\right|_{B T^{\alpha}} \simeq \gamma_{\alpha}$ determine $\gamma_{r}$ uniquely up to homotopy. These considerations lead to our third step.

Step 3. We need to prove the following two equations:

(i) $\lim ^{i+1} \Pi_{i}^{G_{r}}=0$ for all $i \geq 1$.

(ii) $\lim ^{i} \Pi_{i}^{G_{r}}=0$ for all $i \geq 1$.

Since $H_{\mathbb{Q}_{p}^{\wedge}}^{*}(h c(K)) \longrightarrow \prod_{\alpha \in K} H_{\mathbb{Q}_{p}^{\wedge}}^{*}\left(B T^{\alpha}\right)$ is a monomorphism, the completion of Step 1, Step 2 and Step 3(i) will show that $\gamma_{r}$ is a realization of $g_{r}: H_{\mathbb{Q}_{p}^{\wedge}}^{*}\left(B G_{r}\right) \longrightarrow$ $H_{\mathbb{Q}_{p}^{\wedge}}^{*}(h c(K))$. We also want to prove that, up to homotopy, $\gamma_{r}$ is uniquely determined by the cohomological data. Let $\delta_{r}: h c(K) \longrightarrow B G_{r_{p}} \wedge$ be another map such that $H_{\mathbb{Q}_{p}^{\wedge}}^{*}\left(\delta_{r}\right)=g_{r}$. Then $H_{\mathbb{Q}_{p}^{\wedge}}^{*}\left(\left.\gamma_{r}\right|_{B T^{\alpha}}\right)=H_{\mathbb{Q}_{p}^{\wedge}}^{*}\left(\left.\delta_{r}\right|_{B T^{\alpha}}\right)$. If we know that under this condition $\left.\left.\gamma_{r}\right|_{B T^{\alpha}} \simeq \delta_{r}\right|_{B T^{\alpha}}$, then we could conclude by Step 3 that $\gamma_{r} \simeq \delta_{r}$.

Step 4. Let $\gamma_{\alpha}, \delta_{\alpha}: B T^{\alpha} \longrightarrow B G_{r_{p}}^{\wedge}$ be two maps such that $H_{\mathbb{Q}_{p}^{\wedge}}^{*}\left(\gamma_{\alpha}\right)=H_{\mathbb{Q}_{p}^{\wedge}}^{*}\left(\delta_{\alpha}\right)=$ $q_{\alpha} g_{r}$. Then we need to show that $\gamma_{\alpha} \simeq \delta_{\alpha}$.

In our case, Step 4 is a direct consequence of Remark 6.2.

A completion of Step 3(ii) and Step 4 will tell us that, up to homotopy, there is at most one map realizing $g_{r}$.

For the proof of the existence of the desired maps (Theorem 4.6), we need to construct two particular maps, namely $\xi: h c(K) \longrightarrow B U(n)_{p}^{\wedge}$ and $\rho_{\omega}: h c(K) \longrightarrow$ $B S O(2 n)_{p}^{\wedge}$. We will discuss the construction in detail only for the latter case. It can be easily adapted to the first case.

Step 1 for $G_{r}=S O(2 n)$ and $g_{r}=h_{\omega}$. For each face $\alpha=\left\{i_{1}, \ldots, i_{t}\right\} \in K$ we specify classes in the polynomial algebra $\mathbb{Z}[\alpha]$. For $i \leq|\alpha|$ we define $c_{i}(\alpha):=\sigma_{i}\left(v_{i_{1}}, \ldots, v_{i_{t}}\right)$ and $p_{i}(\alpha):=(-1)^{i} \sigma_{i}\left(v_{i_{1}}^{2}, \ldots, v_{i_{t}}^{2}\right)$. If $i>|\alpha|$ we set $c_{i}(\alpha)=p_{i}(\alpha)=0$. We also need an Euler class $e(\alpha)$ defined by $e(\alpha):=c_{n}(\alpha)$ if $|\alpha|=n$ and $e(\alpha)=0$ if $|\alpha|<n$. Here, $\sigma_{i}$ again denotes the $i$-th elementary symmetric polynomial.

Let $\omega: M_{K} \longrightarrow\{ \pm 1\}$ be a function and $e_{\omega}$ the associated square root of $(-1)^{n} p_{n}(K)$. The composition

$$
\mathbb{Z}\left[p_{1}, \ldots, p_{n-1}, e_{n}\right] \stackrel{h_{\omega}}{\longrightarrow} \mathbb{Z}[K] \stackrel{q_{\alpha}}{\longrightarrow} \mathbb{Z}[\alpha]
$$


of $q_{\alpha}$ (defined in Remark 2.11) and $h_{\omega}$ maps $p_{i}$ onto $p_{i}(\alpha)$ and $e_{n}$ onto 0 if $|\alpha|<n$ and onto $\omega(\alpha) e(\alpha)$ if $|\alpha|=n$. This algebraic map can be realized by a composition

$$
\rho_{\omega \alpha}: B T^{\alpha} \longrightarrow B T^{n} \longrightarrow B S O(2 n),
$$

where the second map is induced by the maximal torus inclusion $T^{n} \subset S O(2 n)$ and the first map by a coordinate-wise inclusion $T^{\alpha} \longrightarrow T^{n}$ combined with complex conjugations on some of the coordinates. For $|\alpha|<n$ any number of conjugations is allowed, but for $|\alpha|=n$, we need an odd number of complex conjugations if $\omega(\alpha)=-1$ and an even number otherwise. This establishes a map $\gamma_{\alpha}: B T^{\alpha} \longrightarrow$ $B S O(2 n)_{p}^{\wedge}$ such that $H_{\mathbb{Q}_{p}^{\wedge}}^{*}\left(\gamma_{\alpha}\right)=\left(q_{\alpha} h_{\omega}\right) \otimes \mathbb{Q}_{p}^{\wedge}$ and completes Step 1.

Step 2 for $G_{r}=S O(2 n)$ and $g_{r}=h_{\omega}$. The Weyl group $W_{S O(2 n)}$ of $S O(2 n)$ is isomorphic to $(\mathbb{Z} / 2)^{n-1} \rtimes \Sigma_{n}$, where $\Sigma_{n}$ acts on $T^{n}$ via permutations of the coordinates and $(\mathbb{Z} / 2)^{n-1}$ via even numbers of complex conjugation on coordinates. In fact, for each pair of coordinates, there exists an element in $(\mathbb{Z} / 2)^{n-1}$ which induces complex conjugation exactly on these two coordinates. For $i=1,2$ let $\phi_{i}: T^{\alpha} \longrightarrow T^{n}$ be a coordinate-wise inclusion combined with $l_{i}$ complex conjugations. If $|\alpha|=n$, then there exists $w \in W_{S O(2 n)}$ such that $\phi_{1}=w \phi_{2}$ precisely when $l_{1}$ and $l_{2}$ are congruent modulo 2. Also, if $|\alpha|<n$, then there exists a missing coordinate which we can use to pass from an even number to an odd number of complex conjugations. In this case, there always exists $w \in W_{S O(2 n)}$ such that $\phi_{1}=w \phi_{2}$. This shows that the underlying homomorphisms for different choices of $\rho_{\omega \alpha}$ are conjugate in $S O(2 n)$ and that different choices produce homotopic maps between the classifying spaces.

This argument also shows that if $\alpha \subset \beta$, the triangle

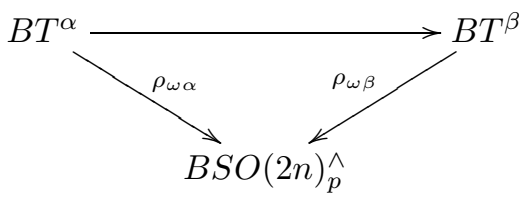

commutes up to homotopy, which completes Step 2.

Example 6.3. We illustrate the above constructions with an example. Let $K$ be the boundary of the 2-dimensional simplex $\Delta[3]$, that is, $\{1,2,3\}$ is the only missing face. Then, the dimension of $K$ is 1 and the Stanley-Reisner algebra is given by $\mathbb{Z}[K]=\mathbb{Z}[3] /\left(v_{1} v_{2} v_{3}\right)$. We fix an Euler class $e_{\omega}:=-v_{1} v_{2}-v_{2} v_{3}+v_{1} v_{3}$ and want to construct a map $\rho: D J(K) \longrightarrow B S O(4)$ such that $\rho^{*}\left(p_{1}\right)=p_{1}(K)=-\left(v_{1}^{2}+v_{2}^{2}+v_{3}^{2}\right)$ and $\rho^{*}\left(e_{1}\right)=e_{\omega}$.

The first factor of the Weyl group, $W_{S O(4)} \cong \mathbb{Z} / 2 \times \mathbb{Z} / 2$, acts on the maximal torus $T^{2}$ by complex conjugation on both coordinates, and the second factor permutes both coordinates. For each $\alpha \in K$ we define a map $\iota_{\alpha}: T^{\alpha} \longrightarrow T^{2}$ as follows. We set $\iota_{\{i\}}\left(z_{i}\right):=\left(z_{i}, 1\right)$ for $i=1,2,3, \iota_{\{1,2\}}\left(z_{1}, z_{2}\right):=\left(\bar{z}_{1}, z_{2}\right), \iota_{\{1,3\}}\left(z_{1}, z_{3}\right):=\left(\bar{z}_{1}, \bar{z}_{3}\right)$ and $\iota_{\{2,3\}}\left(z_{2}, z_{3}\right):=\left(\bar{z}_{2}, z_{3}\right)$. The maps $\rho_{\alpha}$ are given by the $p$-adic completion of the map $B T^{\alpha} \longrightarrow B S O(4)$ induced by the composition of $\iota_{\alpha}$ and the maximal torus inclusion $T^{2} \longrightarrow S O(4)$. For maximal faces $\alpha \in K$ the pull back of the universal Euler and first Pontrjagin class along $\rho_{\alpha}$ are given by $\left(\rho_{\{1,2\}}\right)^{*}\left(e_{2}\right)=-v_{1} v_{2}$, $\left(\rho_{\{1,3\}}\right)^{*}\left(e_{2}\right)=-v_{1} v_{3}$ and $\left(\rho_{\{2,3\}}\right)^{*}\left(e_{2}\right)=v_{2} v_{3}$.

The restriction $\left.\iota_{\{1,3\}}\right|_{T\{3\}}$ and $\iota_{\{3\}}$ differ by a permutation and complex conjugation of both coordinates, and are conjugate in $S O(4)$ and establish homotopic 
maps $\left.\rho_{\{1,3\}}\right|_{B T\{3\}}$ and $\rho_{\{3\}}$. Similarly, for all other choices of $j \in \alpha,|\alpha|=2$, we get $\left.\rho_{\alpha}\right|_{B T\{j\}} \simeq \rho_{\{j\}}$.

Step 1 and Step 2 for $G_{r}=U(n)$ and $g_{r}=f$. In the case of $U(n)$ we do not need to care about complex conjugation. The composition $\mathbb{Z}\left[c_{1}, \ldots, c_{n}\right] \stackrel{f}{\longrightarrow} \mathbb{Z}[K] \stackrel{q_{\alpha}}{\longrightarrow} \mathbb{Z}[\alpha]$ maps $c_{i}$ onto $c_{i}(\alpha)$. The map

$$
\xi_{\alpha}: B T^{\alpha} \longrightarrow B T^{n} \longrightarrow B U(n)_{p}^{\wedge}
$$

is induced by the composition $T^{\alpha} \longrightarrow T^{n} \longrightarrow U(n)$ of some coordinate-wise inclusion followed by the maximal torus inclusion. The Weyl group of $U(n)$ is the symmetric group $\Sigma_{n}$ which acts on $T^{n}$ via permutations of the coordinates. Hence, different coordinate-wise inclusions differ by an element of $\Sigma_{n}$ and induce homotopic maps $B T^{\alpha} \longrightarrow B U(n)_{p}^{\wedge}$.

For $n \leq r$ and a face $\alpha \in K$, we denote by $\gamma_{r \alpha}: B T^{\alpha} \longrightarrow B G_{r}$ one of the maps $\xi_{\alpha} \oplus \mathbb{C}^{r-n}, \rho_{\omega \alpha} \oplus \mathbb{R}^{2(r-n)}$ or $\left(\xi_{\alpha}\right)_{\mathbb{R}} \oplus \mathbb{R}^{2(r-n)+1}$. By the above construction these maps fit together to define an element in $\lim \left[B T^{K}, B G_{r}\right]$ and define functors $\Pi_{i}^{G_{r}}: \operatorname{CAT}(K)^{o p} \longrightarrow \mathrm{AB}$.

Step 3 for all cases. The completion of this step is contained in the more general statement below, namely Proposition 6.4

Proof of Theorem 4.6 and Theorem 4.7. The first theorem follows from the completion of Step 1, Step 2 and Step 3(i) for $g_{r}=f$ and $g_{r}=h_{\omega}$, and the second theorem from the completion of Step 3(ii) and Step 4 for all cases of $g_{r}$ described above.

Proposition 6.4. For all $j \geq i \geq 1$, we have

$$
\lim ^{j} \Pi_{i}^{G_{r}}=0 .
$$

The proof needs some preparation and will cover the rest of this section. The involved mapping spaces can be described in terms of centralizers of subtori. A map $\gamma_{r \alpha}$ is induced by a homomorphism $\iota_{\alpha}: T^{\alpha} \longrightarrow G_{r}$ which is given by the composition of a coordinate-wise inclusion $T^{\alpha} \longrightarrow T_{G(r)}$, possibly some conjugations on coordinates, and the inclusion of the maximal torus into $G(r)$. Conjugation on coordinates of the maximal torus has no effect on the centralizer of $\iota_{\alpha}$. The centralizer $C_{G_{r}}\left(T^{\alpha}\right):=C_{G_{r}}(\alpha)$ of the image of $\iota_{\alpha}$ can be identified with $T^{\alpha} \times G_{r-|\alpha|} \subset G_{r}$, where the inclusions of the factors are induced by the subset relations $\alpha \subset[r]$ and $[r] \backslash \alpha \subset[r]$.

By [N1], there exists a mod- $p$ equivalence

$$
B T^{\alpha} \times B G_{r-|\alpha|}=B C_{G_{r}}\left(j_{\alpha}\right) \longrightarrow \operatorname{map}\left(B T^{\alpha}, B G_{r p}\right)_{\gamma_{\alpha}} .
$$

Moreover, up to homotopy the above mod- $p$ equivalence is natural with respect to morphisms $\beta \supset \alpha$ in $\operatorname{CAT}(K)^{o p}$. Such an inclusion induces the composition

$$
B T^{\beta} \times B G_{r-|\beta|} \longrightarrow B T^{\alpha} \times B T^{\alpha \backslash \beta} \times B G_{r-|\beta|} \longrightarrow B T^{\alpha} \times B G_{r-|\alpha|}
$$

between the classifying spaces of the centralizers. After $p$-adic completion this map is homotopic to the induced map between the associated mapping spaces. Passing to homotopy groups, this describes the map

$$
\Pi_{i}^{G_{r}}(\alpha) \longrightarrow \Pi_{i}^{G_{r}}(\beta) .
$$


It will be convenient to define functors

$$
\Psi_{2}, \hat{\Pi}_{2}^{G_{r}}: \mathrm{CAT}(K)^{o p} \longrightarrow \mathrm{AB}
$$

by $\Psi_{2}(\alpha):=\pi_{2}\left(\left(B T^{\alpha}\right)_{p}^{\wedge}\right)$ and $\hat{\Pi}_{2}^{G}(\alpha):=\pi_{2}\left(B G_{(r-|\alpha|)} \hat{p}\right)$.

In the following we partly need to distinguish between $U(r)$ on the one hand and $S O(2 r)$ and $S O(2 r+1)$ on the other hand. We will denote by $S O_{r}$ one of the special orthogonal groups $S O(2 r)$ or $S O(2 r+1)$.

\section{Lemma 6.5.}

(i) For $i=2$, we have an exact sequence

$$
0 \longrightarrow \hat{\Pi}_{2}^{G_{r}} \longrightarrow \Pi_{2}^{G_{r}} \longrightarrow \Psi_{2} \longrightarrow 0
$$

of functors. Moreover, we have $\Psi_{2} \cong H^{2}\left(B T^{K} ; \mathbb{Z}_{p}^{\wedge}\right)$.

(ii) $\Pi_{2 j+1}^{U(r)}(\alpha)=0$ for all $j \leq r-|\alpha|-1$.

(iii) Let $\alpha \subset \beta$ and $|\beta| \leq n-j$. If $j \geq 2$, then $\Pi_{2 j}^{U(r)}(\beta) \cong \Pi_{2 j}^{U(r)}(\alpha)$. If $j=1$, the same formula holds for $\hat{\Pi}_{2}^{G_{r}}$.

(iv) $\Pi_{1}^{G_{r}}(\alpha)=0$ for all $\alpha$.

(v) If $p$ is odd, then $\hat{\Pi}_{2}^{S O_{r}}(\alpha)=0$ for $|\alpha| \neq r-1$.

(vi) If $t \geq 3$ and $\alpha \subset \beta$, then $\Pi_{t}^{S O_{r}}(\beta) \longrightarrow \Pi_{t}^{S O_{r}}(\alpha)$ is an isomorphism for $|\beta| \leq r-t / 2-1 / 2$. If $p=2$, the same formula holds for $\hat{\Pi}_{2}^{S O_{r}}$.

Proof. The natural transformations $\hat{\Pi}_{2}^{G_{r}} \longrightarrow \Pi_{2}^{G_{r}}$ and $\Pi_{2}^{G_{r}} \longrightarrow \Psi_{2}$ establish the exact sequence of part (i). The second claim of (i) is obvious.

Part (ii) and part (iii) follow from the fact that $\pi_{2 s+1}(B U(t))=0$ for $0 \leq s \leq t-1$ and $\pi_{2 s}(B U(t)) \cong \pi_{2 s}(B U(t+1))$ for $1 \leq s \leq t$.

For every connected compact Lie group $H$, the classifying space $B H$ is simply connected. This proves part (iv).

The fifth part follows from the fact that $\pi_{2}(B S O(s)) \cong \mathbb{Z} / 2$ for $s \geq 3$.

Finally, $\pi_{t}(B S O(k)) \longrightarrow \pi_{t}(B S O(k+1))$ is an isomorphism for $t \leq k-1$. Since $\Pi_{t}^{S O_{r}}(\beta)=\pi_{t}\left(B S O_{n-|\beta| p}\right)$ and $\left.\hat{\Pi}_{2}^{S O_{r}}(\beta)=\pi_{2}\left(B S O_{n-|\beta|}\right)_{p}^{\wedge}\right)$, this implies the last claim.

Proof of Proposition 6.4. Since $\Pi_{1}^{G_{r}} \equiv 0$ (Lemma 6.5(iv)), we have $\lim ^{j} \Pi_{1}^{G_{r}}=0$ for $j \geq 1$.

Now we consider the functor $\Pi_{i}^{U(r)}$ for $i \geq 3$. If $i=2 k+1$ and $|\alpha| \leq n-i+1=$ $n-2 k \leq n-k-1$, then $\Pi_{2 k+1}^{U(r)}(\alpha)=0$ (Lemma 6.5(ii)). Lemma 6.5(iii) implies that $\lim ^{j} \Pi_{i}^{U(r)}=0$ for $j \geq i$.

If $i=2 k \geq 3$ and $|\alpha| \leq n-2 k \leq n-k-1$, then $\Pi_{i}^{U(r)}(\alpha)=\mathbb{Z}_{p}^{\wedge}$ and $\Pi_{i}^{U(r)}(\alpha) \cong$ $\Pi_{i}^{U(r)}(\beta)$ for $\beta \subset \alpha$ by Lemma 6.5(iii). Hence, by Lemma [5.1(iii), we again have $\lim ^{j} \Pi_{i}^{U(r)}=0$ for $j \geq i$.

The same argument shows that $\lim ^{j} \hat{\Pi}_{2}^{U(r)}=0$ for $j \geq 2$.

By Lemma 6.5(i) the functor $\Pi_{2}^{U(r)}$ fits into the exact sequence

$$
0 \longrightarrow \hat{\Pi}_{2}^{U(r)} \longrightarrow \Pi_{2}^{U(r)} \longrightarrow \Psi_{2} \cong H^{2}\left(B T^{K} ; \mathbb{Z}_{p}^{\wedge}\right) \longrightarrow 0 .
$$

For $j \geq 2$, we have $\lim ^{j} H^{2}\left(B T^{K} ; \mathbb{Z}_{p}^{\wedge}\right)=0$ [NR1] as well as $\lim ^{j} \hat{\Pi}_{2}^{U(r)}=0$. Hence, the same holds for $\Pi_{2}^{U(r)}$. This finishes the proof in the complex case. 
Now we consider the cases of the special orthogonal groups. If $j \geq i \geq 3$ we have $n-j+1 \leq n-i+1 \leq n-i / 2-1 / 2$. This shows that $\Pi_{i}^{S O_{r}}(\beta) \cong \Pi_{i}^{S O_{r}}(\alpha)$ for $\alpha \subset \beta$ and $|\beta| \leq n-j+1$ (Lemma 6.5(vi)) and that $\lim ^{j} \Pi_{i}^{S O_{r}}=0$ (Lemma 5.1(iii)).

For $i=2$ we again first consider the functor $\hat{\Pi}_{2}^{S O_{r}}$. If $p$ is odd, then $\hat{\Pi}_{2}^{S O_{r}}(\alpha) \neq 0$ if and only if $|\alpha|=r-1$ and $S O_{r}=S O(2 r)$. Hence, if $r \geq n+2$ or $S O_{r}=S O(2 r+1)$, the functor $\hat{\Pi}_{2}^{S O_{r}}$ is trivial and $\lim ^{j} \hat{\Pi}_{2}^{S O_{r}}=0$. For $r=n, n+1$ and $S O_{r}=S O(2 r)$, the functor $\hat{\Pi}_{2}^{S O_{r}}$ is a product of atomic functors and only non-trivial either on faces of order $n$ or $n-1$. But in both cases we have $\lim ^{j} \hat{\Pi}_{2}^{S O_{r}}=0$ for $j \geq 2$ (Lemma $5.1(\mathrm{i}))$.

If $p=2$, then

$$
\hat{\Pi}_{2}^{S O_{r}}(\alpha) \cong \pi_{2}\left(B S O_{r-|\alpha|_{2}^{\wedge}}\right) \cong \begin{cases}0 & \text { for } r=|\alpha|, \\ \mathbb{Z}_{2}^{\wedge} & \text { for } r=|\alpha|+1, S O_{r}=S O(2 r), \\ \mathbb{Z} / 2 & \text { otherwise. }\end{cases}
$$

If $r \geq n+1$ or if $r \geq n$ and $S O_{r}=S O(2 r+1)$, we have $\hat{\Pi}_{2}^{S O_{r}}(\beta) \cong \hat{\Pi}_{2}^{S O_{r}}(\alpha)$ for $\alpha \subset \beta$ and $|\beta| \leq n-2+1$. Using Lemma 5.1(iii) again, this shows that in these cases $\lim ^{j} \hat{\Pi}_{2}^{S O_{r}}=0$ for $j \geq 2$. If $r=n$, then we have an exact sequence of functors

$$
0 \longrightarrow \Phi \longrightarrow \hat{\Pi}_{2}^{S O(2 r)} \longrightarrow \hat{\Pi}_{2}^{S O(2 r+1)} \longrightarrow 0
$$

where the natural transformation $\hat{\Pi}_{2}^{S O(2 r)} \longrightarrow \hat{\Pi}_{2}^{S O(2 r+1)}$ is induced by the inclusion $S O(2 r) \rightarrow S O(2 r+1)$ and is an epimorphism. For the kernel $\Phi$ we have $\Phi=$ $\Phi_{n-1}$, which implies that $\lim ^{s} \Phi=0$ for $s \neq 1$ (Lemma 5.1(i)). This shows that $\lim ^{j} \hat{\Pi}_{2}^{S O(2 r)}=0$ for $j \geq 2$ as well.

Now, the same argument as for $\Pi_{2}^{U(r)}$ shows that $\lim ^{j} \Pi_{2}^{S O_{r}}=0$. This finishes the proof for $\mathrm{SO}_{r}$.

\section{Complex structures for vector Bundles}

The stable uniqueness result for vector bundles over $D J(K)$ realizing the total Pontrjagin class $p(K)=\prod_{i}\left(1-v_{i}^{2}\right)$ shows that all the bundles $\rho_{\omega}: D J(K) \longrightarrow$ $B S O(2 n)$ of Theorem 4.2 admit stably a complex structure. In fact, $\rho_{\omega} \oplus \mathbb{R}^{2} \cong$ $\xi_{\mathbb{R}} \oplus \mathbb{R}^{2}$ already admits a complex structure. In this section we will discuss the unstable version, namely which of the bundles $\rho_{\omega}$ actually are isomorphic to the realification of a complex vector bundle. We also will classify the complex structures of $\rho_{\omega}$ up to isomorphism, in the unstable case as well as in the stable case. For this purpose we need to classify all complex bundles $\eta$ over $D J(K)$ with $p\left(\eta_{\mathbb{R}}\right)=p(K)$.

For every function $f:[m] \longrightarrow\{ \pm 1\}$ we define an element

$$
c_{f}(K):=\prod_{i=1}^{m}\left(1+f(i) v_{i}\right) \in \mathbb{Z}[K]=H^{*}(D J(K) ; \mathbb{Z}) .
$$

We will construct a complex vector bundle $\xi_{f} \downarrow D J(K)$ with $c\left(\xi_{f}\right)=c_{f}(K)$. If $f(i)=1$ for all $i \in[m]$, then $\xi_{f}=\xi$.

For each $\alpha \in K$ we define a homomorphism $\iota_{f, \alpha}: T^{\alpha} \longrightarrow T^{\alpha}$ which is given by complex conjugation on the $i$-th coordinate if $f(i)=-1$ and the identity otherwise. The collection of the maps $\theta_{f, \alpha}: B T^{\alpha} \longrightarrow B T^{\alpha}$ induced by $\iota_{f, \alpha}$ provides a natural 
transformation $\Theta_{f}: B T^{K} \longrightarrow B T^{K}$ and therefore a map

$$
\theta_{f}: D J(K) \simeq c(K) \longrightarrow c(K) \simeq D J(K) .
$$

By construction, the square $\theta_{f}^{2}$ is homotopic to the identity on $D J(K)$. In cohomology, the induced map $\theta_{f}^{*}: \mathbb{Z}[K] \longrightarrow \mathbb{Z}[K]$ maps $v_{i}$ to $f(i) v_{i}$. The total Chern class of the composition $\xi_{f}:=\xi \theta_{f}: D J(K) \longrightarrow B U(n)$ is given by $c\left(\xi_{f}\right)=$ $\prod_{i=1}^{m}\left(1+f(i) v_{i}\right)=c_{f}(K)$, the Euler class of $\left(\xi_{f}\right)_{\mathbb{R}}$ by $e\left(\left(\xi_{f}\right)_{\mathbb{R}}\right)=c_{n}\left(\xi_{f}\right)=$ $\sum_{\mu \in M_{K}} \omega_{f}(\mu) v_{\mu}=e_{f}(K)$ and the total Pontrjagin class by $p\left(\left(\xi_{f}\right)_{\mathbb{R}}\right)=p\left(\xi_{f}\right)=$ $p(K)$. As already explained in the introduction, the function $\omega_{f}: M_{K} \longrightarrow\{ \pm 1\}$ is given by $\omega_{f}(\mu)=\prod_{i \in \mu} f(i)$.

Proposition 7.1. Let $f, g:[m] \longrightarrow\{ \pm 1\}$ be two functions.

(i) The complex vector bundles $\xi_{f}$ and $\xi_{g}$ are isomorphic if and only if $f=g$.

(ii) The realifications $\left(\xi_{f}\right)_{\mathbb{R}}$ and $\left(\xi_{g}\right)_{\mathbb{R}}$ are isomorphic as non-oriented bundles if and only if $\omega_{f}= \pm \omega_{g}$.

Proof. If $\xi_{f} \cong \xi_{g}$, then both have the same Chern classes given by $\prod_{i}\left(1+f(i) v_{i}\right)$ and $\prod_{i}\left(1+g(i) v_{i}\right)$. A comparison of the first Chern class of both bundles already shows that $f(i)=g(i)$ for all $i$.

Since the Euler classes of $\xi_{f}$ and $\xi_{g}$ are given by $e_{f}(K)$ and $e_{g}(K)$, the bundles $\xi_{f}$ and $\xi_{g}$ are isomorphic as non-oriented real vector bundles if and only if the Euler classes of both bundles differ at most by a sign (Theorem 1.2 and Remark 1.5). Also, this is true if and only if $\omega_{f}= \pm \omega_{g}$.

The next theorem classifies all complex bundles whose realification realizes the Pontrjagin class $p(K)=\prod_{i}\left(1-v_{i}^{2}\right)$.

Theorem 7.2. Let $K$ be an $(n-1)$-dimensional simplicial complex and $\eta$ : $D J(K)$ $\longrightarrow B U(n)$ a complex vector bundle.

(i) For any map $f:[m] \longrightarrow\{ \pm 1\}$ the map $\eta$ is homotopic to $\xi_{f}$ if and only if $H^{*}(\eta ; \mathbb{Q})=H^{*}\left(\xi_{f} ; \mathbb{Q}\right)$.

(ii) If $p\left(\eta_{\mathbb{R}}\right)=p(K)$, then there exists a function $f:[m] \longrightarrow\{ \pm 1\}$ such that $\eta \simeq \xi_{f}$.

Proof. The first part follows from the fact that $\theta_{f}^{2}=i d_{D J(K)}$. If $\eta$ and $\xi_{f}$ induce the same map in rational cohomology, then the same holds for $\eta \theta_{f}$ and $\xi_{f} \theta_{f} \simeq \xi \theta_{f}^{2} \simeq \xi$. Hence, by Theorem 1.2, both maps are homotopic as well as $\eta$ and $\xi_{f}$.

Now let $\eta: D J(K) \longrightarrow B U(n)$ be a complex vector bundle with $p\left(\eta_{\mathbb{R}}\right)=$ $\prod_{i=1}^{m}\left(1-v_{i}^{2}\right)$. The Euler class $e\left(\eta_{\mathbb{R}}\right)$ is a square root of $(-1)^{n} p_{n}(K)$ and determines a function $\omega: M_{K} \longrightarrow\{ \pm 1\}$ such that $e\left(\eta_{\mathbb{R}}\right)=e_{\omega}(K)$. By Theorem 4.3, the maps $\eta_{\mathbb{R}}$ and $\rho_{\omega}$ are homotopic.

For each $\alpha \in K$, the composition

$$
\eta_{\alpha}: B T^{\alpha} \longrightarrow D J(K) \stackrel{\eta}{\longrightarrow} B U(n)
$$

is homotopic to a map induced by a homomorphism $\iota_{\alpha}: T^{\alpha} \longrightarrow T^{n}$ composed with the maximal torus inclusion $T^{n} \longrightarrow U(n)$ (Theorem 6.1). Also, the composition

$$
B T^{\alpha} \longrightarrow D J(K) \stackrel{\eta}{\longrightarrow} B U(n) \longrightarrow B S O(2 n)
$$

is homotopic to a map induced by $\iota_{\alpha}$ composed with $T^{n} \longrightarrow U(n) \longrightarrow S O(2 n)$. By construction, the restriction $\left.\rho_{\omega}\right|_{B T^{\alpha}}: B T^{\alpha} \longrightarrow B S O(2 n)$ is homotopic to a map induced by a coordinate-wise inclusion $T^{\alpha} \longrightarrow T^{n}$ followed by complex conjugation 
on some coordinates and composed with $T^{n} \longrightarrow S O(2 n)$. We can again apply Theorem 6.1. This shows that $\iota_{\alpha}: T^{\alpha} \longrightarrow T^{n}$ is of the same form, namely a coordinate-wise inclusion followed by complex conjugation on some coordinates. If $\beta \subset \alpha$, the composition $\left.\iota_{\alpha}\right|_{T^{\beta}}: T^{\beta} \longrightarrow T^{\alpha} \stackrel{\iota_{\alpha}}{\longrightarrow} T^{n}$ and the homomorphism $\iota_{\beta}: T^{\beta} \longrightarrow T^{n}$ differ only by an element of the Weyl group of $U(n)$, that is, by a permutation of the coordinates. Such an operation has no effect on complex conjugations. This allows us to define a map $f:[m] \longrightarrow\{ \pm 1\}$ by $f(i)=-1$ if the homomorphism $\iota_{\{i\}}: T^{\{i\}} \longrightarrow T^{n}$ involves complex conjugation and $f(i)=1$ otherwise.

By construction of the function $f$, we have $\left.\left.\xi_{f}\right|_{B T^{\alpha}} \simeq \eta\right|_{B T^{\alpha}}$ for all $\alpha \in K$ (Theorem 6.1). In particular, the maps $\eta$ and $\xi_{f}$ induce the same map in rational cohomology and are therefore homotopic (part (i)).

Remark 7.3. Proposition 7.1 and Theorem 7.2 also have stable versions. The same arguments as above work. Suppose that $t \geq 1$.

(i) For two functions $f, g:[m] \longrightarrow\{ \pm 1\}$, the bundles $\xi_{f} \oplus \mathbb{C}^{t}$ and $\xi_{g} \oplus \mathbb{C}^{t}$ are isomorphic if and only if $f=g$, but the realifications $\left(\xi_{f}\right)_{\mathbb{R}} \oplus \mathbb{R}^{2 t}$ and $\left(\xi_{g}\right)_{\mathbb{R}} \oplus \mathbb{R}^{2 t}$ are always isomorphic.

(ii) Let $\eta: D J(K) \longrightarrow B U(n+t)$ be an $(n+t)$-dimensional complex vector bundle. Then, $\eta$ and $\xi_{f} \oplus \mathbb{C}^{t}$ are homotopic if and only if both induce the same map in rational cohomology. Also, if $p\left(\eta_{\mathbb{R}}\right)=p(K)$, then there exists a function $f:[m] \longrightarrow\{ \pm 1\}$ such that $\eta \cong \xi_{f} \oplus \mathbb{C}^{t}$.

Now we are in the position to prove Theorem 1.4 and Corollary 1.7, both of which we recall for the convenience of the reader.

Theorem 1.4. Let $K$ be an $(n-1)$-dimensional simplicial complex. Let $\rho: D J(K)$ $\longrightarrow B S O(2 n)$ be an oriented real vector bundle such that $p(\rho)=p(K)$. Then, the vector bundle $\rho$ admits a complex structure if and only if there exists a function $f:[m] \longrightarrow\{ \pm 1\}$ such that $e(\rho)= \pm e_{f}(K)$.

Corollary 1.7. Let $K$ be an $(n-1)$-dimensional abstract finite simplicial complex and let $s \geq n$. Let $\rho$ be a $2 s$-dimensional oriented real vector bundle over $D J(K)$ such that $p(\rho)=p(K)$.

(i) If $s=n$, the number of isomorphism classes of complex structures for $\rho$ equals the number of functions $f:[m] \longrightarrow\{ \pm 1\}$ such that $e_{f}(K)= \pm e(\rho)$. In particular, there exists only a finite number of isomorphism classes of complex structures for $\rho$.

(ii) If $s>n$, then there exist exactly $2^{m}$ non-isomorphic complex structures for $\rho$.

Proof of Theorem 1.4. An even-dimensional orientable real vector bundle $\rho$ over $D J(K)$ admits two orientations. Also, switching the orientation means to multiply the Euler class by -1 . We denote the bundle with the opposite orientation by $\bar{\rho}$. In particular, $\rho$ and $\bar{\rho}$ are isomorphic as non-oriented real vector bundles.

If the Euler class $e(\rho)$ of $\rho$ has the required form, then either $\rho$ or $\bar{\rho}$ and $\left(\xi_{f}\right)_{\mathbb{R}}$ have the same Euler class and the same Pontrjagin classes. By Theorem 4.3, this shows that either $\rho \simeq\left(\xi_{f}\right)_{\mathbb{R}}$ or that $\bar{\rho} \simeq\left(\xi_{f}\right)_{\mathbb{R}}$. In particular, $\rho$ admits a complex structure.

If $\rho$ admits a complex structure, then there exists a complex vector bundle $\eta: D J(K) \longrightarrow B U(n)$ such that $\rho$ and $\eta_{\mathbb{R}}$ are isomorphic as non-oriented bundles. 
In particular, $p\left(\eta_{\mathbb{R}}\right)=p(\rho)=\prod_{i}\left(1-v_{i}^{2}\right)$. By Theorem 7.2 , there exists a function $f:[m] \longrightarrow\{ \pm 1\}$ such that $\eta \simeq \xi_{f}$. Also, this implies that $e(\rho)= \pm e\left(\left(\xi_{f}\right)_{\mathbb{R}}\right)=$ $\pm e_{f}(K)$.

Proof of Corollary 1.7. First we assume that $s=n$. If $\eta$ is a complex structure for $\rho$, then, by Theorem 7.2 , there exists a function $f:[m] \longrightarrow\{ \pm 1\}$ such that $\eta \cong \xi_{f}$. Moreover, we have $e_{f}(K)=e\left(\eta_{\mathbb{R}}\right)= \pm e(\rho)$. Also, two functions $f, g:[m] \longrightarrow\{ \pm 1\}$ induce isomorphic vector bundles $\xi_{f}$ and $\xi_{g}$ if and only if $f=g$ (Proposition 7.1). This proves the first part.

If $s>n$, then, for every function $f:[m] \longrightarrow\{ \pm 1\}$, the two bundles $\left(\xi_{f}\right)_{\mathbb{R}} \oplus$ $\mathbb{R}^{2(s-n)}$ and $\rho$ have the same Pontrjagin classes and are isomorphic (Theorem 1.2). Moreover, Remark 7.3 tells us that every complex structure of $\rho$ is of the form $\xi_{f} \oplus \mathbb{C}^{s-n}$ and that $\xi_{f} \oplus \mathbb{C}^{s-n} \cong \xi_{g} \oplus \mathbb{C}^{s-n}$ if and only if $f=g$. This completes the proof of the second part.

Examples 7.4. We illustrate our results with some examples. If $K:=\partial \Delta[3]$ is a triangle, we have three vertices, three maximal faces given by $:=\{1,2\},\{2,3\}$ and $\{1,3\}$, and eight choices for the Euler class $e_{\omega}(K)$, namely $\pm v_{1} v_{2} \pm v_{2} v_{3} \pm v_{1} v_{3}$. If the number of negative signs is even, then there exists a function $f:[3] \longrightarrow\{ \pm 1\}$ such that $e_{\omega}(K)=\omega_{f}(\{1,2\}) v_{1} v_{2}+\omega_{f}(\{2,3\}) v_{2} v_{3}+\omega_{f}(\{1,3\}) v_{1} v_{3}=c_{3}\left(\xi_{f}\right)$. In this case, $\rho_{\omega} \cong\left(\xi_{f}\right)_{\mathbb{R}}$ as oriented bundles. Since $\operatorname{dim} K$ is odd, faces of maximal order contain an even number of vertices and $e_{f}(K)=e_{-f}(K)$. In particular, there exist two different complex structures for $\rho_{\omega}$ in this case. If the number of signs in $e_{\omega}(K)$ is odd, such a function does not exist and $\rho_{\omega}$ is not isomorphic to the realification of a complex bundle as an oriented bundle. Since the number of maximal faces is odd, one of the two orientations on $\rho$ satisfies the condition on the signs and every bundle $\rho_{\omega}$ always admits exactly two non-isomorphic complex structures.

If $K$ is a square, we have four vertices and four maximal faces given by $\{1,2\}$, $\{2,3\},\{3,4\}$ and $\{1,4\}$. Every function $f:[4] \longrightarrow\{ \pm 1\}$ produces an Euler class $\pm v_{1} v_{2} \pm v_{2} v_{3} \pm v_{3} v_{4} \pm v_{1} v_{4}$ with an even number of negative signs. Again, $f$ and $-f$ produce the same Euler class. Since the number of maximal faces is even, the Euler classes for the two orientations on $\rho_{\omega}$ involve both either an even or an odd number of negative signs. We have 16 choices for the Euler class, where eight of them admit exactly two non-isomorphic complex structures and eight of them do not admit a complex structure.

If $K=\partial \Delta[4]$, then we have 4 maximal faces, 4 vertices, 16 possible Euler classes $\pm v_{1} v_{2} v_{3} \pm v_{1} v_{2} v_{4} \pm v_{1} v_{3} v_{4} \pm v_{2} v_{3} v_{4}$ and 16 non-isomorphic bundles $\xi_{f}$. An easy calculation shows that, in this case, the map $f \mapsto \omega_{f}$ is injective and surjective. Hence, every $\omega: M_{K} \longrightarrow\{ \pm 1\}$ is of the form $\omega_{f}$ and every $\rho$ admits exactly two complex structures.

Now we assume that $K$ is a complex of dimension $n-1$ and admits an $n$ coloring $g: K \longrightarrow \Delta[n]$. The composition $f g$ of a function $f:[n] \longrightarrow\{ \pm 1\}$ and the restriction $\left.g\right|_{[m]}$ of $g$ to the set of vertices $[m]$ produces the Euler class $e_{\omega}=\epsilon \sum_{\mu} v_{\mu}$ where $\epsilon:=\prod_{i \in[n]} f(i)$. This shows that in this case, the bundle $\xi_{\mathbb{R}}$ admits at least $2^{n}$ non-isomorphic complex structures.

Remark 7.5. For a simple polytope $P$ we denote by $K_{P}$ the dual of the boundary of $P$. Since $P$ is simple, $K_{P}$ is a simplicial polytope, in particular a simplicial complex, homeomorphic to $S^{n-1}$. An orientation for $P$ or $K_{P}$ inherits an orientation to 
every maximal face $\mu \in K_{P}$, and hence, an orientation on the set $\mu$. For a matrix $\Lambda \in \mathbb{Z}^{n \times m}$ and a maximal face $\mu \in K_{P}$ we denote by $\Lambda_{\mu}$ the maximal minor given by the columns of $\Lambda$ associated to the vertices in $\mu$, but we order the columns according to the orientation of $\mu$. That is, the order of the columns is only fixed up to even permutations, but such a permutation will not change the determinant.

A dicharacteristic pair $(P, \Lambda)$ consists of an oriented simple polytope $P$ of dimension $n$ with $m$ facets and a matrix $\Lambda \in \mathbb{Z}^{n \times m}$ such that $\operatorname{det} A_{\mu}= \pm 1$ for all maximal faces of $K_{P}$. Up to diffeomorphism, every oriented quasi-toric manifold can be constructed from a dicharacteristic pair $(P, \Lambda)$ (e.g. see $[\mathrm{BP})$. The Euler class of $\left(\tau_{M}\right)_{h T^{n}}$ is then given by $e\left(\left(\tau_{M}\right) h T^{n}\right)=\sum_{\mu \in M_{K_{P}}} \operatorname{det} \Lambda_{\mu} v_{\mu}$ [DN]. In particular, the real vector bundle $\left(\tau_{M}\right)_{h T^{n}}$ admits a complex structure if and only if their exists a function $f:[m] \longrightarrow\{ \pm 1\}$ and $\epsilon= \pm 1$ such that $\operatorname{det} A_{\mu}=\epsilon f(\mu)$ for all maximal faces $\mu \in K_{P}$. This is exactly the condition sufficient and necessary for the existence of an almost complex structure for $M^{2 n}$ (e.g. see BP, Corollary $5.54]$ and the following remarks).

If we stabilize the bundle $\left(\tau_{M}\right)_{h T^{n}}$, the picture will change. Since $\left(\tau_{M}\right)_{h T^{n}} \oplus \mathbb{R}^{2} \cong$ $\xi_{\mathbb{R}} \oplus \mathbb{R}^{2}$ (Theorem 1.2), Corollary 1.7 gives a complete list of the isomorphism classes of complex structures for $\left(\tau_{M}\right)_{h T^{n}} \oplus \mathbb{R}^{2 s}$ for $s \geq 1$.

\section{ACKNOWLEDGEMENT}

The author would like to express his gratitude to Natalia Dobrinskaya, Taras Panov and Nigel Ray for many helpful discussions.

\section{REFERENCES}

[AM] J.F. Adams and Z. Mahmud, Maps between classifying spaces, Inv. Math. 35 (1976), 1-41. MR0423352 (54:11331)

[BBCG] A. Bahri, M. Bendersky, F.R. Cohen and S. Gitler, Decompositions of the polyhedral product functor with applications to moment-angle complexes anf related spaces, Proc. Natl. Acad. Sci. USA 106 (2009), 12241-12244. MR2539227 (2010j:57036)

[BK] A.K. Bousfield and D.M. Kan, Homotopy Limits, Completions and Localizations, Volume 304 of Lecture Notes in Mathematics, Springer Verlag (1972). MR0365573 (51:1825)

[BP] V.M. Buchstaber and T.E. Panov, Torus Actions and Their Applications in Topology and Combinatorics, volume 24 of University Lecture Series, American Mathematical Society (2002). MR1897064 (2003e:57039)

[DJ] M.W. Davis and T. Januszkiewicz, Convex polytopes, Coxeter orbifolds and torus actions, Duke Math. J. 62 (1991), 417-451. MR1104531 (92i:52012)

[DN] N. Dobrinskaja and D. Notbohm, Equivariant almost complex structures for quasi-toric manifolds, in preparation.

[DW] W.G. Dwyer and C.W. Wilkerson, Homotopy fixed-point methods for Lie groups and finite loop spaces, Ann. Math. (2) 139 (1994), 395-442. MR.1274096 (95e:55019)

$[\mathrm{K}] \quad$ A. Kustarev, Quasitoric manifolds with invariant almost complex structure, Preprint (2009).

[N1] D. Notbohm, Maps between classifying spaces, Math. Z. 207 (1991), 153-168. MR1106820 (92b:55017)

[N2] D. Notbohm, Colorings of simplicial complexes and vector bundles over DavisJanuszkiewicz spaces, Math. Z. 266 (2010), no. 2, 399-405. MR2678634

[NR1] D. Notbohm and N. Ray, On Davis-Januszkiewicz homotopy types. I. Formality and Rationalisation, Algebr. Geom. Topol. 5 (2005), 31-51. MR2135544 (2006a:55016)

[NR2] D. Notbohm and N. Ray, On Davis-Januszkiewicz homotopy types. II. Completion and Globalisation, to appear in Algebr. Geom. Topol. MR2683752

[O] R. Oliver, Higher limits via Steinberg representations, Comm. in Algebra 22 (1994), 1381-1393. MR1261265 (95b:18007) 
[S] G. Segal, Equivariant K-theory, Publ. Math., Inst. Hautes Ètud. Sci. 34 (1968), 129-151. MR0234452 (38:2769)

[V] R.M. Vogt, Convenient categories of topological spaces for homotopy theory, Arch. Math. 22 (1971), 545-555. MR0300277 (45:9323)

[W] Z. Wojtkowiak, On maps from holim F to Z, Algebraic Topology, Barcelona 1986, Volume 1298 of Lecture Notes in Mathematics, Springer Verlag (1987). MR.928836 (89a:55034)

Department of Mathematics, Faculty of Sciences, Vrije Universiteit, De Boolelaan 1081A, 1081 HV Amsterdam, The Netherlands

E-mail address: notbohm@few.vu.nl 